\title{
Ledipasvir and sofosbuvir plus ribavirin in patients with genotype 1 or 4 hepatitis $C$ virus infection and advanced liver disease: a multicentre, open-label, randomised, phase 2 trial
}

\author{
Michael Manns, Didier Samuel, EdwardJ Gane, David Mutimer, Geoff McCaughan, Maria Buti, Martín Prieto, José Luis Calleja, \\ Markus Peck-Radosavljevic, Beat Müllhaupt, Kosh Agarwal, Peter Angus, Eric M Yoshida, Massimo Colombo, Mario Rizzetto, Hadas Dvory-Sobol, \\ Jill Denning, Sarah Arterburn, Phillip S Pang, Diana Brainard, John G McHutchison, Jean-François Dufour, Hans Van Vlierberghe, Bart van Hoek, \\ Xavier Forns, for the SOLAR-2 investigators
}

\section{Summary}

Background Treatment options are limited for patients infected by hepatitis C virus (HCV) with advanced liver disease. We assessed the safety and efficacy of ledipasvir, sofosbuvir, and ribavirin in patients with HCV genotype 1 or 4 and advanced liver disease.

Methods We did an open-label study at 34 sites in Europe, Canada, Australia, and New Zealand. Cohort A included patients with Child-Turcotte-Pugh class B (CTP-B) or CTP-C cirrhosis who had not undergone liver transplantation. Cohort B included post-transplantation patients who had either no cirrhosis; CTP-A, CTP-B, or CTP-C cirrhosis; or fibrosing cholestatic hepatitis. Patients in each group were randomly assigned (1:1) using a computer-generated randomisation sequence to receive 12 or 24 weeks of ledipasvir $(90 \mathrm{mg})$ and sofosbuvir $(400 \mathrm{mg})$ once daily (combination tablet), plus ribavirin (600-1200 mg daily). The primary endpoint was the proportion of patients achieving a sustained virological response 12 weeks after treatment (SVR12). All patients who received at least one dose of study drug were included in the safety analysis and all patients who received at least one dose of study drug and did not undergo liver transplantation during treatment were included in the efficacy analyses. Estimates of SVR12 and relapse rates and their two-sided $90 \%$ CI (Clopper-Pearson method) were provided. This exploratory phase 2 study was not powered for formal comparisons among treatment groups; no statistical hypothesis testing was planned or conducted. The trial is registered with EudraCT (number 2013-002802-30) and ClinicalTrials.gov (number NCT02010255).

Findings Between Jan 14, 2014, and Aug 19, 2014, 398 patients were screened. Of 333 patients who received treatment, 296 had genotype $1 \mathrm{HCV}$ and 37 had genotype $4 \mathrm{HCV}$. In cohort A, among patients with genotype $1 \mathrm{HCV}$, SVR12 was achieved by 20 (87\%, 90\% CI 70-96) of 23 CTP-B patients with 12 weeks of treatment; 22 (96\%, 81-100) of 23 CTP-B patients with 24 weeks of treatment; 17 (85\%, 66-96) of 20 CTP-C patients (12 weeks treatment); and 18 (78\%, 60-91) of 23 CTP-C patients (24 weeks treatment). In cohort B, among patients with genotype 1 HCV, SVR12 was achieved by 42 (93\%, 84-98) of 45 patients without cirrhosis (12 weeks treatment); 44 (100\%, 93-100) of 44 patients without cirrhosis (24 weeks treatment); 30 (100\%, 91-100) of 30 CTP-A patients (12 weeks treatment); 27 (96\%, 84-100) of 28 CTP-A patients (24 weeks treatment); 19 (95\%, 78-100) of 20 CTP-B patients (12 weeks treatment); 20 (100\%, 86-100) of 20 CTP-B patients (24 weeks treatment); one (50\%, 3-98) of two CTP-C patients (12 weeks treatment); and four (80\%, 34-99) of five CTP-C patients (24 weeks treatment). All five patients with fibrosing cholestatic hepatitis achieved SVR12 (100\%, 90\% CI 55-100). Among all patients with genotype 4 HCV, SVR12 was achieved by 14 (78\%, 56-92) of 18 patients (12 weeks treatment) and 16 (94\%, 75-100) of 17 patients (24 weeks treatment). Seven patients (2\%) discontinued ledipasvir-sofosbuvir prematurely due to adverse events. 17 patients died, mainly from complications of hepatic decompensation.

Interpretation Ledipasvir-sofosbuvir and ribavirin provided high rates of SVR12 for patients with advanced liver disease, including those with decompensated cirrhosis before or after liver transplantation.

Funding Gilead Sciences.

\section{Introduction}

Patients with chronic hepatitis C virus (HCV) infection with advanced fibrosis or cirrhosis are at increased risk of hepatocellular carcinoma, liver failure, liver transplantation, and both liver-related and all-cause mortality. ${ }^{1}$ For patients who undergo liver transplantation with detectable HCV, recurrent infection is universal. In some transplantation patients, recurrent HCV infection leads to an aggressive course of disease known as fibrosing cholestatic hepatitis, which is characterised by increased risk of cirrhosis, hepatic decompensation, and death. ${ }^{2}$ Despite the serious risks for patients with $\mathrm{HCV}$ with advanced liver disease, treatment options for these patients are limited.

The nucleotide analogue sofosbuvir is a direct-acting inhibitor of the HCV NS5B polymerase and was approved in the USA in 2013 and in Europe in early 2014. ${ }^{3,4}$ In
Lancet Infect Dis 2016; 16: 685-97

Published Online February 18, 2016 http://dx.doi.org/10.1016/ S1473-3099(16)00052-9 This online publication has been corrected. The corrected version first appeared at thelancet.com/ infection on May 23, 2016

See Comment page 624

Department of Gastroenterology, Hepatology and Endocrinology, Hannover Medical School, Hannover, Germany, and German Center of Infection Research (DZIF), Hannover-Braunschweig, Germany (Prof M Manns MD); Centre Hepatobiliaire, Hôpital Paul Brousse, and Université Paris Sud, Villejuif, France (Prof D Samuel MD); New Zealand Liver Transplant Unit, Auckland City Hospital, Auckland, New Zealand (Prof EJ Gane MD); Queen Elizabeth Hospital and NIHR Liver Biomedical Research Unit, Birmingham, UK

(Prof D Mutimer MD), Australian Liver Transplant Unit, Centenary Research Institute, Royal Prince Alfred Hospital, University of Sydney, Sydney, Australia (Prof G McCaughan MD), Internal Medicine and Hepatology, CIBERHED Vall d'Hebron University Hospital, Barcelona, Spain (Prof M Buti MD); Digestive Diseases Service, Hospital Universitario y Politécnico La Fe, and Centro de Investigación Biomédica en Red de Enfermedades Hepáticas y Digestivas Valencia, Spain (M Prieto MD); Servicio de Gastroenterología y Hepatología Hospital Universitario Puerta de Hierro Universidad Autonoma, 
Madrid, Spain (J L Calleja MD); Department of Gastroenterology and Hepatology, Medical University of Vienna, Vienna, Austria (Prof M Peck-Radosavljevic MD); Department for Gastroenterology and Hepatology UniversitätsSpital Zürich, Zürich, Switzerland (Prof B Müllhaupt MD); Institute of Liver Studies, King's College Hospital Foundation Trust, London, UK (K Agarwal MD); Liver Transplant Unit, Austin

Hospital, Heidelberg, VIC, Australia (Prof P Angus MD); Division of Gastroenterology,

Vancouver General Hospital and University of British Columbia, Vancouver, BC, Canada (E M Yoshida MD); Department of

Gastroenterology, IRCCS Maggiore Hospital University of Milan, Milan, Italy (Prof M Colombo MD); Azienda Ospedaliero-Universitaria, Torino, Italy

(Prof M Rizzetto MD); Gilead Sciences, Foster City, CA, USA (H Dvory-Sobol PhD, J Denning MA, S Arterburn MS, PS Pang MD, D Brainard MD, J G McHutchison MD);

Department of Hepatology,

University of Bern, Bern, Switzerland (J-F Dufour MD); Ghent University Hospital, Ghent, Belgium

(Prof H Van Vlierberghe MD); Department of

Gastroenterology and Hepatology, Leiden University Medical Centre, Leiden, Netherlands (Prof B van Hoek MD); and Liver Unit, Hospital Clinic, CIBEREHD, IDIBAPS, University of Barcelona, Barcelona, Spain (X Forns MD)

Correspondence to: Prof Michael Manns, Department of Gastroenterology, Hepatology and Endocrinology, Hannover Medical School, Carl Neuberg Strasse 1, 30625 Hannover, Germany

manns.michael@mhhannover.de

See Online for appendix

\section{Research in context}

\section{Evidence before this study}

This study was designed in the first half of 2013, before any alloral regimens for hepatitis C virus (HCV) had been approved. We did PubMed searches for articles using the search terms "HCV treatment", "decompensated cirrhosis", and "post-liver transplant" from inception until Feb 20, 2013. There were no language restrictions for this search. We found no reports on the use of direct-acting antivirals in all-oral regimens for HCV in patients with advanced fibrosis or decompensated cirrhosis.

\section{Added value of this study}

This study assessed the safety and efficacy of the all-oral regimen of ledipasvir, sofosbuvir, and ribavirin in patients with HCV who have advanced liver disease or have had a liver transplant. The results show that ledipasvir-sofosbuvir plus ribavirin for 12 weeks is an effective treatment for patients with advanced liver disease, including those with decompensated liver function before and after liver transplantation. These patients, who are at substantial risk for mortality, had no effective treatment options until recently. Extending treatment to 24 weeks did not appear to be associated with improved outcomes.

phase 2 trials, sofosbuvir plus ribavirin was effective in treating patients before and after liver transplantation, including those with compensated cirrhosis. ${ }^{5-7}$ The combination of sofosbuvir and ribavirin was given for 24-48 weeks, which might be cumbersome for patients with advanced disease who could have difficulty tolerating the haematological side-effects associated with ribavirin.

In 2014, a fixed-dose combination of sofosbuvir with the HCV NS5A inhibitor ledipasvir was approved in the USA for treating chronic genotype $1 \mathrm{HCV}$ infection and in Europe for treating genotypes 1 or 4 infection. ${ }^{8,9}$ In a phase 2 study of $\mathrm{HCV}$ genotype 1 patients with compensated cirrhosis, the combination of sofosbuvir and ledipasvir for 24 weeks led to a sustained virological response (SVR) rate of $97 \% .^{10}$ In the US-based SOLAR-1 study, ${ }^{11}$ the combination of sofosbuvir, ledipasvir, and ribavirin for 12 or 24 weeks had moderate-to-high SVR rates in $\mathrm{HCV}$ patients, predominantly with HCV genotype 1a, who had advanced liver disease, including those who had undergone liver transplantation. Here we present the results of the international SOLAR-2 study, in which we similarly assessed the safety and efficacy of sofosbuvir, ledipasvir, and ribavirin for 12 or 24 weeks in HCV genotype 1a, 1b, or 4 patients with advanced liver disease, including post-transplantation patients with fibrosing cholestatic hepatitis. This phase 2 study was exploratory and not powered to permit comparisons among groups; no statistical hypothesis testing was planned or conducted.

\section{Methods}

\section{Study design and participants}

We did an open-label, phase 2 study at 34 clinical sites in Europe, Canada, Australia, and New Zealand (six in
Implications of all the available evidence

Similar results were recorded in the SOLAR-1 study, which also assessed ledipasvir, sofosbuvir, and ribavirin treatment in patients with advanced liver disease before and after liver transplantation, but which enrolled almost exclusively patients with genotype $1 \mathrm{HCV}$ (99\%). The recent CORAL-1 study showed that $97 \%$ of patients with mild histological recurrent HCV after liver transplantation achieved sustained virological response after treatment with the combination of ombitasvir-paritaprevir-ritonavir and dasabuvir plus ribavirin for 24 weeks. The three studies meet a crucial need of assessing novel therapies in patients who have limited treatment options. Our findings support the efficacy and safety of ledipasvir-sofosbuvir plus ribavirin in patients with decompensated liver disease before and after liver transplantation, and suggest that there is no clinically significant interaction between ledipasvir-sofosbuvir and common immunosuppressive agents used in liver transplant recipients.

Canada, four in France, four in Germany, four in Spain, three in the UK, two in Australia, two in Austria, two in Belgium, two in Italy, two in the Netherlands, two in Switzerland, and one in New Zealand). Eligible patients were at least 18 years old and chronically infected with genotype 1 or $4 \mathrm{HCV}$.

Patients were enrolled in two cohorts. In both cohorts, the presence of cirrhosis was established by either liver biopsy, FibroScan scores greater than $12.5 \mathrm{kPa}$, or FibroTest scores greater than 0.75 , and an aspartate aminotransferase to platelet ratio of more than 2 . Cohort A consisted of patients who had not undergone liver transplantation and had decompensated cirrhosis with either moderate hepatic impairment (ChildTurcotte-Pugh class B [CTP-B]; group 1) or severe hepatic impairment (CTP-C; group 2). Cohort B consisted of five groups of patients who had previously undergone liver transplantation: group 3 patients did not have cirrhosis group 4 patients had compensated cirrhosis and mild hepatic impairment (CTP-A), group 5 patients had decompensated cirrhosis and moderate hepatic impairment (CTP-B), group 6 patients had decompensated cirrhosis and severe hepatic impairment (CTP-C), and group 7 patients had fibrosing cholestatic hepatitis. In both cohorts, enrolment of patients with CTP-C class hepatic impairment was restricted to patients with scores of $10-12$ points (appendix).

Patients in cohort B, groups 3-6 had to have undergone liver transplantation at least 3 months before initiation of study treatment. Patients in cohort B, group 7 had to have undergone liver transplantation at least 2 months, but not more than 18 months, before initiation of study treatment and have histological evidence of fibrosing 
cholestatic hepatitis in the previous 6 months, bilirubin at least 2.5 times the upper limit of normal, and no evidence of hepatic artery thrombosis in the previous 6 months. Also for this group, any patients with alternative explanations for their cholestasis or hyperbilirubinaemia, such as biliary or hepatic artery complications or drug-induced injury, would not be eligible.

Patients co-infected with HIV or hepatitis B virus or those with previous exposure to an NS5A inhibitor were excluded. Patients could not have had treatment with interferon, ribavirin, telaprevir, boceprevir, or any other HCV drugs within 1 month before screening. Additional details on exclusion criteria can be found in the appendix.

The design of this study, which was in compliance with the principles of the Declaration of Helsinki, Good Clinical Practice guidelines, and local regulatory requirements, was approved by the institutional review board or independent ethics committee at each participating site. The study was done according to the protocol of the funder (Gilead Sciences) in collaboration with the academic investigators.

\section{Randomisation}

Patients in each of the seven groups were randomly assigned using a computer-generated randomisation sequence generated by Bracket (San Francisco, CA, USA) and allocated by means of an interactive web response system (1:1 ratio) to receive either 12 or 24 weeks of treatment with ledipasvir $90 \mathrm{mg}$ and sofosbuvir $400 \mathrm{mg}$ in a fixed-dose combination tablet (ledipasvir-sofosbuvir) once daily plus ribavirin.

\section{Procedures}

For groups 1, 2, 5, and 6, ribavirin was given at a starting dose of $600 \mathrm{mg}$ per day divided morning and evening. If the starting dose was well tolerated and haemoglobin concentrations remained above $10 \mathrm{~g} / \mathrm{dL}$ without haematological growth factor support, the dose could be increased to $1000 \mathrm{mg}$ daily in patients weighing less than $75 \mathrm{~kg}$ and $1200 \mathrm{mg}$ daily in patients weighing at least $75 \mathrm{~kg}$. For patients who could not tolerate the starting dose of $600 \mathrm{mg}$, the dose was reduced as necessary. For groups 3,4 , and 7 , ribavirin was given orally twice daily via weight-based dosing (1000 mg per day for patients weighing less than $75 \mathrm{~kg}$ and $1200 \mathrm{mg}$ per day for patients weighing at least $75 \mathrm{~kg}$ ).

Management of immunosuppression was not specified in the clinical protocol, but treatments and dose adjustments were recorded to assess any possible effect on the resolution of HCV infection. Patients who underwent liver transplantation during the study discontinued all study drugs about $24 \mathrm{~h}$ before transplantation.

Serum HCV RNA was measured using the COBAS AmpliPrep/COBAS Taqman HCV Test, version 2.0, for use with the High Pure System (Roche Molecular Diagnostics, Pleasanton, CA, USA). HCV genotype and subtype were identified using the VERSANT HCV Genotype INNO-LiPA 2.0 assay, the TRUGENE HCV 5'NC Genotyping Assay (Siemens, Munich, Germany), and NS5B sequencing. $I L-28 B$ genotype was determined by PCR amplification of the single-nucleotide polymorphism rs12979860.

For analysis of viral resistance, samples were collected at baseline from all patients. The HCV NS5A and NS5B coding regions were amplified using standard RT-PCR. Ultradeep sequencing was done. Variants present at more than $1 \%$ of sequence reads were reported. For patients who had virological failure, sequencing was again done for the HCV NS5A and NS5B coding regions, with samples collected at the first virological failure timepoint. Variants in NS5A and NS5B coding regions present in at least $1 \%$ of the viral population were compared with the respective baseline sequence.

\section{Outcomes}

The primary efficacy endpoint was the proportion of patients achieving an SVR 12 weeks after the end of therapy (SVR12), defined as serum HCV RNA below the lower limit of quantification (LLOQ, $15 \mathrm{IU} / \mathrm{mL}$ ). Secondary endpoints included improvements in CTP and Model for End-stage Liver Disease (MELD) scores at 12 weeks after treatment for all patients who achieved SVR12. The primary safety endpoint was the proportion of patients who discontinued study treatment because of an adverse event.

\section{Statistical analysis}

All patients who received at least one dose of study drugs, met criteria for one of the seven disease groups, and did not have an on-study transplant while on treatment or within 70 days post-treatment with HCV RNA below the LLOQ at last measurement before transplantation were included in the primary efficacy analysis. For each group and treatment duration, the percentages of patients with SVR12 were calculated along with a two-sided 90\% CI (using the Clopper-Pearson method). We randomised the assignment of patients to 12 or 24 weeks of treatment within each of the seven disease groups to ensure that an equal number of patients received both durations of treatment within each disease group and that patient assignment within disease group was random. No hypothesis testing of the primary efficacy outcome was planned or done.

Except for group 3, all groups had an enrolment target of 50 patients. The enrolment target for group 3 (posttransplantation without cirrhosis) was 100 patients. The enrolment targets were not based on a formal sample size calculation but were believed to provide a reasonable estimate of SVR12 with appropriate $90 \%$ CIs. No formal comparisons between groups or between treatment durations within each group were planned.

Patients who underwent liver transplantation from day 1 to day 70 after treatment (the beginning of the 


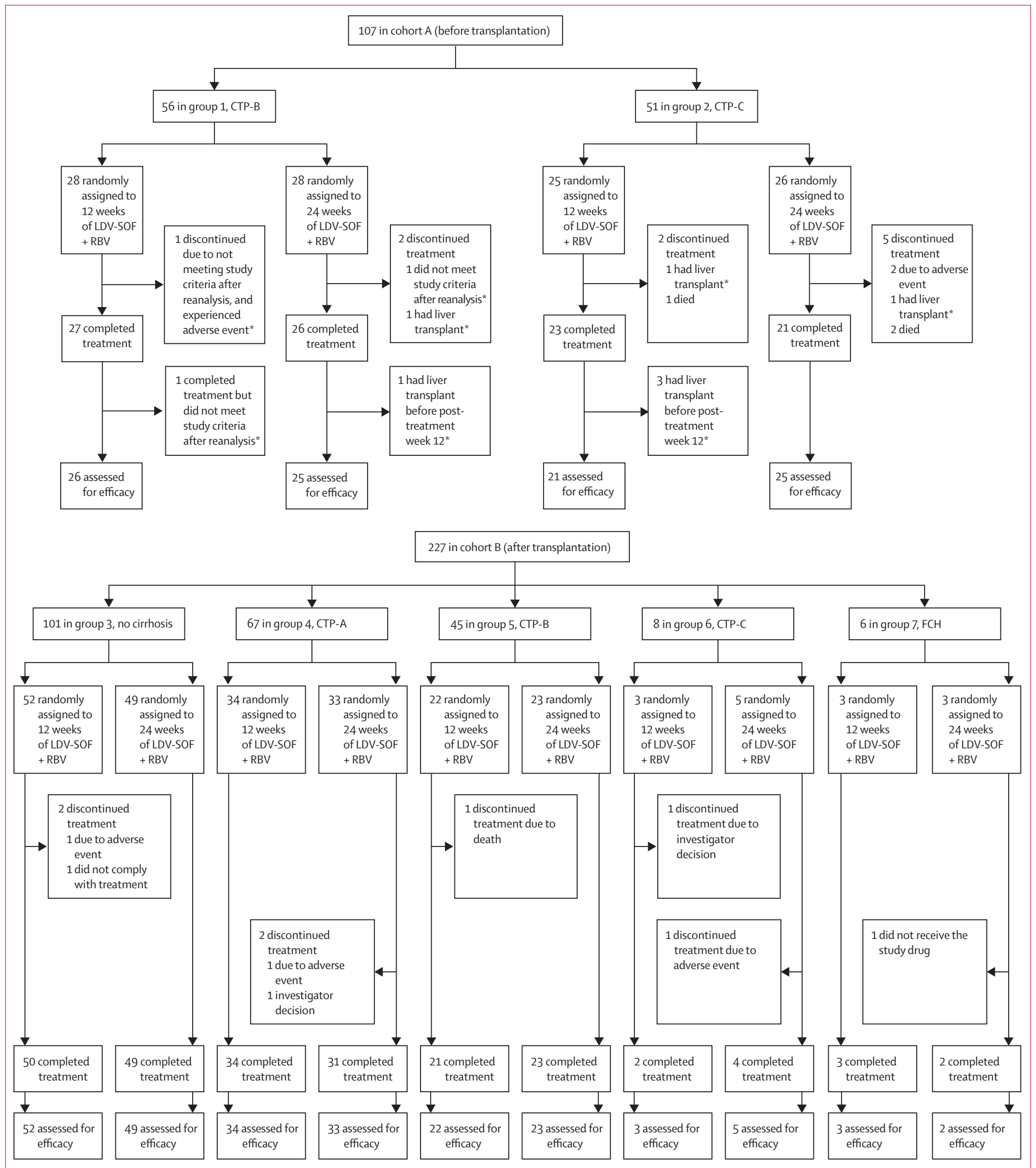


post-treatment week 12 visit window) with HCV RNA less than $15 \mathrm{IU} / \mathrm{mL}$ at last measurement before transplantation were excluded from the numerator and denominator for the primary efficacy analysis (SVR12). Instead, efficacy in these patients was assessed in a separate analysis. For all patients who received a transplant on study with HCV RNA less than $15 \mathrm{IU} / \mathrm{mL}$ at their last measurement before transplantation, the rate of post-transplantation virological response (pTVR12, defined as HCV RNA < $15 \mathrm{IU} / \mathrm{mL} 12$ weeks after date of transplantation) was calculated.

\section{Role of the funding source}

The funder collected the data, monitored the conduct of the study, and did the statistical analyses. An independent data and safety monitoring committee reviewed the progress of the study. The investigators, participating institutions, and funder agreed to maintain confidentiality of the data. All authors had access to the data and assume responsibility for the integrity and completeness of the data and analyses reported. The first draft of the manuscript was prepared by a professional writer paid by Gilead Sciences and the corresponding author, with input from all the authors.

\section{Results}

Between Jan 14, 2014, and Aug 19, 2014, 398 patients were screened, of which 333 received at least one dose of study treatment (figure 1, appendix). Of these 333 patients, $296(89 \%)$ patients had genotype $1 \mathrm{HCV}$ infection $(n=163$ genotype $1 \mathrm{a}$ and $n=133$ genotype $1 \mathrm{~b})$, and $37(11 \%)$ had genotype $4 \mathrm{HCV}$ infection (table 1). Among 227 patients enrolled with cirrhosis, 67 had CTP-A liver disease, 101 had CTP-B liver disease, and 59 had CTP-C liver disease. Of the 226 patients who had undergone liver transplantation before enrolment, 101 had Metavir F0-F3 fibrosis, 67 had CTP-A liver disease, 45 had CTP-B liver disease, eight had CTP-C liver disease, and five had fibrosing cholestatic hepatitis. In each of the treatment groups, patients were predominantly white and male, and most had previously received treatment for HCV.

Among patients with genotype $1 \mathrm{HCV}$ and CTP-B disease who had not undergone transplantation, SVR12 was achieved by 20 (87\%, 90\% CI 70-96) of 23 patients receiving 12 weeks of treatment and 22 (96\%, 81-100) of 23 patients receiving 24 weeks of treatment (table 2). For patients with genotype $1 \mathrm{HCV}$ and CTP-C disease, SVR12 was achieved by 17 (85\%, 66-96) of 20 patients receiving 12 weeks of treatment and by 18 (78\%, 60-91) of 23 patients receiving 24 weeks of treatment. In total,

Figure 1: Trial profile

CTP=Child-Turcotte-Pugh. FCH=fibrosing cholestatic hepatitis. LDV=ledipasvir RBV=ribavirin. SOF=sofosbuvir. *Patients excluded from the full analysis set used to analyse efficacy data: seven patients who received liver transplants before post-treatment week 12; and three patients who did not meet criteria for one of seven treatment groups after reanalysis (patients had not received a liver transplant nor were their screened CTP scores greater than or equal to 7).
12 patients did not achieve SVR12: six had virological relapse (four in the 12-week group and two in the 24-week group), five died (two in the 12-week group and three in the 24-week group), and one patient in the 24-week group withdrew consent. Six patients underwent liver transplantation before SVR12 with HCV RNA less than the LLOQ at transplantation (four in the 12-week group, two in the 24-week group). All six of these patients still had HCV RNA less than LLOQ 12 weeks posttransplantation.

Among HCV genotype 1 liver transplant recipients without cirrhosis, SVR12 was achieved by 42 (93\%, 84-98) of 45 patients receiving 12 weeks of treatment and $44(100 \%, 93-100)$ of 44 patients receiving 24 weeks of treatment. In liver transplant recipients with CTP-A disease, SVR12 was achieved by $30(100 \%, 91-100)$ of 30 patients receiving 12 weeks of treatment and 27 (96\%, $84-100)$ of 28 patients receiving 24 weeks of treatment. In total, four patients did not achieve SVR12: one noncirrhotic patient receiving 12 weeks of treatment had a relapse, and three died (two non-cirrhotic patients receiving 12 weeks of treatment and one with CTP-A cirrhosis receiving 24 weeks of treatment). None of the deaths were considered related to study drug.

In liver transplant recipients with genotype $1 \mathrm{HCV}$ and CTP-B cirrhosis, SVR12 was achieved by 19 (95\%, 78-100) of 20 patients receiving 12 weeks of treatment and 20 $(100 \%, 86-100)$ of 20 patients receiving 24 weeks of treatment (table 2). Of the two patients with CTP-C cirrhosis who received 12 weeks of treatment, one achieved SVR12. Of the five patients with CTP-C cirrhosis who received 24 weeks of treatment, four achieved SVR12. Three transplant recipients with CTP-B or CTP-C cirrhosis did not achieve SVR12 because they died.

All five patients with fibrosing cholestatic hepatitisthree receiving 12 weeks of treatment and two receiving 24 weeks of treatment-achieved SVR12.

Estimates $(95 \% \mathrm{CI})$ of SVR12 and relapse rates for the 12-week and 24-week groups and the difference (95\% CI on difference) between groups did not show any statistically significant differences $(95 \%$ CI on difference included 0) between the 12-week and 24-week groups for SVR12 or virological relapse (data not shown). However, the groups were not powered for such comparisons so this analysis does not show equivalent efficacy of the 12-week and 24-week treatment durations.

For analysis of relapse rates, patients who were transplanted before post-treatment week 12 with last HCV RNA less than LLOQ before transplantation and patients with other virological outcomes (eg, deaths, withdrawn consent) were excluded from the analysis. Five $(3 \%)$ of 150 genotype 1a patients versus two $(2 \%)$ of 126 genotype $1 \mathrm{~b}$ patients relapsed $(\mathrm{p}=0 \cdot 46$, two-sided Fisher's exact test).

Among the 35 patients with genotype $4 \mathrm{HCV}$ who were assessed for efficacy, SVR12 was achieved in 14 (78\%, $90 \%$ CI 56-92) of 18 patients receiving 12 weeks of 


\begin{tabular}{|c|c|c|c|c|c|c|c|c|c|c|c|c|c|c|}
\hline & \multicolumn{4}{|c|}{ Cohort A: before transplantation } & \multicolumn{10}{|c|}{ Cohort B: after transplantation } \\
\hline & \multicolumn{2}{|c|}{ Group 1, CTP-B } & \multicolumn{2}{|c|}{ Group 2, CTP-C } & \multicolumn{2}{|c|}{ Group 3, no cirrhosis } & \multicolumn{2}{|c|}{ Group 4, CTP-A } & \multicolumn{2}{|c|}{ Group 5, CTP-B } & \multicolumn{2}{|c|}{ Group 6, CTP-C } & \multicolumn{2}{|c|}{ Group 7, FCH } \\
\hline & $\begin{array}{l}12 \text { weeks } \\
(\mathrm{n}=28)\end{array}$ & $\begin{array}{l}24 \text { weeks } \\
(n=28)\end{array}$ & $\begin{array}{l}12 \text { weeks } \\
(n=25)\end{array}$ & $\begin{array}{l}24 \text { weeks } \\
(n=26)\end{array}$ & $\begin{array}{l}12 \text { weeks } \\
(n=52)\end{array}$ & $\begin{array}{l}24 \text { weeks } \\
(n=49)\end{array}$ & $\begin{array}{l}12 \text { weeks } \\
(n=34)\end{array}$ & $\begin{array}{l}24 \text { weeks } \\
(n=33)\end{array}$ & $\begin{array}{l}12 \text { weeks } \\
(n=22)\end{array}$ & $\begin{array}{l}24 \text { weeks } \\
(n=23)\end{array}$ & $\begin{array}{l}12 \text { weeks } \\
(n=3)\end{array}$ & $\begin{array}{l}24 \text { weeks } \\
(n=5)\end{array}$ & $\begin{array}{l}12 \text { weeks } \\
(n=3)\end{array}$ & $\begin{array}{l}24 \text { weeks } \\
(\mathrm{n}=2)\end{array}$ \\
\hline $\begin{array}{l}\text { Median age } \\
\text { (years) }\end{array}$ & $\begin{array}{l}57 \\
(52-61)\end{array}$ & $\begin{array}{l}57 \\
(52-62)\end{array}$ & $\begin{array}{l}58 \\
(53-63)\end{array}$ & $\begin{array}{l}50 \\
(47-62)\end{array}$ & $\begin{array}{c}58 \\
(54-64)\end{array}$ & $\begin{array}{c}60 \\
(54-63)\end{array}$ & $\begin{array}{c}58 \\
(55-61)\end{array}$ & $\begin{array}{c}62 \\
(58-67)\end{array}$ & $\begin{array}{l}58 \\
(52-61)\end{array}$ & $\begin{array}{l}60 \\
(56-65)\end{array}$ & $\begin{array}{l}63 \\
(57-66)\end{array}$ & $\begin{array}{l}62 \\
(54-65)\end{array}$ & $\begin{array}{l}57 \\
(56-61)\end{array}$ & $\begin{array}{l}56 \\
(54-58)\end{array}$ \\
\hline Males & $\begin{array}{l}23 \\
(82 \%)\end{array}$ & $\begin{array}{l}19 \\
(68 \%)\end{array}$ & $\begin{array}{l}15 \\
(60 \%)\end{array}$ & $\begin{array}{l}20 \\
(77 \%)\end{array}$ & $\begin{array}{c}41 \\
(79 \%)\end{array}$ & $\begin{array}{c}39 \\
(80 \%)\end{array}$ & $\begin{array}{c}28 \\
(82 \%)\end{array}$ & $\begin{array}{c}26 \\
(79 \%)\end{array}$ & $\begin{array}{l}15 \\
(68 \%)\end{array}$ & $\begin{array}{l}15 \\
(65 \%)\end{array}$ & $\begin{array}{l}2 \\
(67 \%)\end{array}$ & $\begin{array}{l}5 \\
(100 \%)\end{array}$ & $\begin{array}{c}2 \\
(67 \%)\end{array}$ & $\begin{array}{c}1 \\
(50 \%)\end{array}$ \\
\hline \multicolumn{15}{|l|}{ Race } \\
\hline White & $\begin{array}{l}25 \\
(89 \%)\end{array}$ & $\begin{array}{l}28 \\
(100 \%)\end{array}$ & $\begin{array}{l}23 \\
(92 \%)\end{array}$ & $\begin{array}{l}25 \\
(96 \%)\end{array}$ & $\begin{array}{c}50 \\
(96 \%)\end{array}$ & $\begin{array}{c}47 \\
(96 \%)\end{array}$ & $\begin{array}{c}33 \\
(97 \%)\end{array}$ & $\begin{array}{c}30 \\
(91 \%)\end{array}$ & $\begin{array}{l}21 \\
(95 \%)\end{array}$ & $\begin{array}{l}21 \\
(91 \%)\end{array}$ & $\begin{array}{c}3 \\
(100)\end{array}$ & $\begin{array}{l}5 \\
(100 \%)\end{array}$ & $\begin{array}{c}2 \\
(67 \%)\end{array}$ & $\begin{array}{c}1 \\
(50 \%)\end{array}$ \\
\hline Black & $\begin{array}{c}1 \\
(4 \%)\end{array}$ & 0 & $\begin{array}{c}1 \\
(4 \%)\end{array}$ & $\begin{array}{c}1 \\
(4 \%)\end{array}$ & $\begin{array}{r}1 \\
(2 \%)\end{array}$ & 0 & 0 & 0 & 0 & $\begin{array}{c}1 \\
(4 \%)\end{array}$ & 0 & 0 & 0 & 0 \\
\hline Other & $\begin{array}{l}2 \\
(7 \%)\end{array}$ & 0 & $\begin{array}{c}1 \\
(4 \%)\end{array}$ & 0 & $\begin{array}{r}1 \\
(2 \%)\end{array}$ & $\begin{array}{c}2 \\
(4 \%)\end{array}$ & $\begin{array}{r}1 \\
(3 \%)\end{array}$ & $\begin{array}{c}3 \\
(9 \%)\end{array}$ & $\begin{array}{c}1 \\
(5 \%)\end{array}$ & $\begin{array}{c}1 \\
(4 \%)\end{array}$ & 0 & 0 & $\begin{array}{c}1 \\
(33 \%)\end{array}$ & $\begin{array}{c}1 \\
(50 \%)\end{array}$ \\
\hline \multicolumn{15}{|l|}{ HCV genotype } \\
\hline $1 \mathrm{a}$ & $\begin{array}{l}13 \\
(46 \%)\end{array}$ & $\begin{array}{l}12 \\
(43 \%)\end{array}$ & $\begin{array}{l}13 \\
(52 \%)\end{array}$ & $\begin{array}{l}12 \\
(46 \%)\end{array}$ & $\begin{array}{c}27 \\
(52 \%)\end{array}$ & $\begin{array}{c}29 \\
(59 \%)\end{array}$ & $\begin{array}{c}14 \\
(41 \%)\end{array}$ & $\begin{array}{c}13 \\
(39 \%)\end{array}$ & $\begin{array}{l}11 \\
(50 \%)\end{array}$ & $\begin{array}{l}13 \\
(57 \%)\end{array}$ & $\begin{array}{c}1 \\
(33 \%)\end{array}$ & $\begin{array}{c}1 \\
(20 \%)\end{array}$ & $\begin{array}{c}2 \\
(67 \%)\end{array}$ & $\begin{array}{c}2 \\
(100 \%)\end{array}$ \\
\hline $1 \mathrm{~b}$ & $\begin{array}{l}12 \\
(43 \%)\end{array}$ & $\begin{array}{l}13 \\
(46 \%)\end{array}$ & $\begin{array}{l}11 \\
(44 \%)\end{array}$ & $\begin{array}{l}11 \\
(42 \%)\end{array}$ & $\begin{array}{c}18 \\
(35 \%)\end{array}$ & $\begin{array}{c}15 \\
(31 \%)\end{array}$ & $\begin{array}{c}16 \\
(47 \%)\end{array}$ & $\begin{array}{r}15 \\
(45)\end{array}$ & $\begin{array}{c}9 \\
(41 \%)\end{array}$ & $\begin{array}{l}7 \\
(30 \%)\end{array}$ & $\begin{array}{c}1 \\
(33 \%)\end{array}$ & $\begin{array}{c}4 \\
(80 \%)\end{array}$ & $\begin{array}{c}1 \\
(33 \%)\end{array}$ & 0 \\
\hline 4 & $\begin{array}{l}3 \\
(11 \%)\end{array}$ & $\begin{array}{l}3 \\
(11 \%)\end{array}$ & $\begin{array}{c}1 \\
(4 \%)\end{array}$ & $\begin{array}{c}3 \\
(12 \%)\end{array}$ & $\begin{array}{c}7 \\
(13 \%)\end{array}$ & $\begin{array}{c}5 \\
(10 \%)\end{array}$ & $\begin{array}{c}4 \\
(12 \%)\end{array}$ & $\begin{array}{c}5 \\
(15 \%)\end{array}$ & $\begin{array}{l}2 \\
(9 \%)\end{array}$ & $\begin{array}{c}3 \\
(13 \%)\end{array}$ & $\begin{array}{c}1 \\
(33 \%)\end{array}$ & 0 & 0 & 0 \\
\hline $\begin{array}{l}\text { HCV RNA }\left(\log _{10}\right. \\
I U / m L)\end{array}$ & $\begin{array}{c}6 \cdot 0 \\
(0 \cdot 5)\end{array}$ & $\begin{array}{c}5.9 \\
(0.6)\end{array}$ & $\begin{array}{c}5 \cdot 6 \\
(0.6)\end{array}$ & $\begin{array}{l}5.7 \\
(0.4)\end{array}$ & $\begin{array}{c}6 \cdot 4 \\
(0 \cdot 7)\end{array}$ & $\begin{array}{c}6.5 \\
(0.4)\end{array}$ & $\begin{array}{l}6.3 \\
(0.6)\end{array}$ & $\begin{array}{l}6.5 \\
(0.6)\end{array}$ & $\begin{array}{c}6 \cdot 1 \\
(0 \cdot 8)\end{array}$ & $\begin{array}{c}6 \cdot 2 \\
(0 \cdot 9)\end{array}$ & $\begin{array}{c}6 \cdot 0 \\
(0 \cdot 5)\end{array}$ & $\begin{array}{c}6.5 \\
(0.5)\end{array}$ & $\begin{array}{c}7 \cdot 3 \\
(0.7)\end{array}$ & $\begin{array}{l}6.0 \\
(0.4)\end{array}$ \\
\hline $\begin{array}{l}\text { HCV RNA } \\
\geq 800000 \mathrm{IU} / \mathrm{mL}\end{array}$ & $\begin{array}{l}17 \\
(61 \%)\end{array}$ & $\begin{array}{l}14 \\
(50 \%)\end{array}$ & $\begin{array}{l}9 \\
(36 \%)\end{array}$ & $\begin{array}{c}9 \\
(35 \%)\end{array}$ & $\begin{array}{c}40 \\
(77 \%)\end{array}$ & $\begin{array}{c}44 \\
(90 \%)\end{array}$ & $\begin{array}{c}25 \\
(74 \%)\end{array}$ & $\begin{array}{c}28 \\
(85 \%)\end{array}$ & $\begin{array}{l}17 \\
(77 \%)\end{array}$ & $\begin{array}{l}14 \\
(61 \%)\end{array}$ & $\begin{array}{c}1 \\
(33 \%)\end{array}$ & $\begin{array}{l}5 \\
(100 \%)\end{array}$ & $\begin{array}{c}3 \\
(100 \%)\end{array}$ & $\begin{array}{c}1 \\
(50 \%)\end{array}$ \\
\hline $\begin{array}{l}\text { IL-28B } \\
\text { genotype CC }\end{array}$ & $\begin{array}{l}6 \\
(21 \%)\end{array}$ & $\begin{array}{l}9 \\
(32 \%)\end{array}$ & $\begin{array}{l}7 \\
(28 \%)\end{array}$ & $\begin{array}{c}4 \\
(15 \%)\end{array}$ & $\begin{array}{c}9 \\
(17 \%)\end{array}$ & $\begin{array}{c}10 \\
(20 \%)\end{array}$ & $\begin{array}{c}3 \\
(9 \%)\end{array}$ & $\begin{array}{c}7 \\
(21 \%)\end{array}$ & $\begin{array}{l}3 \\
(14 \%)\end{array}$ & $\begin{array}{l}5 \\
(22 \%)\end{array}$ & 0 & $\begin{array}{l}3 \\
(60 \%)\end{array}$ & 0 & $\begin{array}{c}1 \\
(50 \%)\end{array}$ \\
\hline Previously treated & $\begin{array}{l}25 \\
(89 \%)\end{array}$ & $\begin{array}{l}24 \\
(86 \%)\end{array}$ & $\begin{array}{l}13 \\
(52 \%)\end{array}$ & $\begin{array}{l}18 \\
(69 \%)\end{array}$ & $\begin{array}{c}41 \\
(79 \%)\end{array}$ & $\begin{array}{c}36 \\
(73 \%)\end{array}$ & $\begin{array}{c}31 \\
(91 \%)\end{array}$ & $\begin{array}{c}29 \\
(88 \%)\end{array}$ & $\begin{array}{l}18 \\
(82 \%)\end{array}$ & $\begin{array}{l}19 \\
(83 \%)\end{array}$ & $\begin{array}{l}2 \\
(67 \%)\end{array}$ & $\begin{array}{l}5 \\
(100 \%)\end{array}$ & $\begin{array}{c}2 \\
(67 \%)\end{array}$ & $\begin{array}{c}2 \\
(100 \%)\end{array}$ \\
\hline \multicolumn{15}{|l|}{$\begin{array}{l}\text { Regimen } \\
\text { received }\end{array}$} \\
\hline PEG/ribavirin & $\begin{array}{l}19 / 25 \\
(76 \%)\end{array}$ & $\begin{array}{l}20 / 24 \\
(83 \%)\end{array}$ & $\begin{array}{c}9 / 13 \\
(69 \%)\end{array}$ & $\begin{array}{l}13 / 18 \\
(72 \%)\end{array}$ & $\begin{array}{l}35 / 41 \\
(85 \%)\end{array}$ & $\begin{array}{l}24 / 36 \\
(67 \%)\end{array}$ & $\begin{array}{l}22 / 31 \\
(71 \%)\end{array}$ & $\begin{array}{l}20 / 29 \\
(69 \%)\end{array}$ & $\begin{array}{r}9 / 18 \\
(50 \%)\end{array}$ & $\begin{array}{l}14 / 19 \\
(74 \%)\end{array}$ & $\begin{array}{l}2 / 2 \\
(100 \%)\end{array}$ & $\begin{array}{l}3 / 5 \\
(60 \%)\end{array}$ & $\begin{array}{c}2 / 2 \\
(100 \%)\end{array}$ & $\begin{array}{c}2 / 2 \\
(100 \%)\end{array}$ \\
\hline $\begin{array}{l}\mathrm{PI}+\mathrm{PEG} / \\
\text { ribavirin }\end{array}$ & $\begin{array}{r}4 / 25 \\
(16 \%)\end{array}$ & $\begin{array}{r}3 / 24 \\
(13 \%)\end{array}$ & $\begin{array}{c}3 / 13 \\
(23 \%)\end{array}$ & $\begin{array}{l}3 / 18 \\
(17 \%)\end{array}$ & $\begin{array}{l}5 / 41 \\
(12 \%)\end{array}$ & $\begin{array}{l}10 / 36 \\
(28 \%)\end{array}$ & $\begin{array}{c}7 / 31 \\
(23 \%)\end{array}$ & $\begin{array}{l}6 / 29 \\
(21 \%)\end{array}$ & $\begin{array}{r}2 / 18 \\
(11 \%)\end{array}$ & $\begin{array}{r}3 / 19 \\
(16 \%)\end{array}$ & 0 & 0 & 0 & 0 \\
\hline Other* & $\begin{array}{l}2 / 25 \\
(8 \%)\end{array}$ & $\begin{array}{l}1 / 24 \\
(4 \%)\end{array}$ & $\begin{array}{l}1 / 13 \\
(8 \%)\end{array}$ & $\begin{array}{l}2 / 18 \\
(11 \%)\end{array}$ & $\begin{array}{l}1 / 41 \\
(2 \%)\end{array}$ & $\begin{array}{l}2 / 36 \\
(6 \%)\end{array}$ & $\begin{array}{l}2 / 31 \\
(6 \%)\end{array}$ & $\begin{array}{l}3 / 29 \\
(10 \%)\end{array}$ & $\begin{array}{r}7 / 18 \\
(39 \%)\end{array}$ & $\begin{array}{r}2 / 19 \\
(11 \%)\end{array}$ & 0 & $\begin{array}{l}2 / 5 \\
(40 \%)\end{array}$ & 0 & 0 \\
\hline \multicolumn{15}{|l|}{$\begin{array}{l}\text { Previous } \\
\text { response }\end{array}$} \\
\hline $\begin{array}{l}\text { Non- } \\
\text { response }\end{array}$ & $\begin{array}{l}20 / 24 \\
(83 \%)\end{array}$ & $\begin{array}{l}13 / 23 \\
(57 \%)\end{array}$ & $\begin{array}{l}10 / 12 \\
(83 \%)\end{array}$ & $\begin{array}{l}11 / 18 \\
(61 \%)\end{array}$ & $\begin{array}{l}28 / 41 \\
(68 \%)\end{array}$ & $\begin{array}{l}16 / 35 \\
(46 \%)\end{array}$ & $\begin{array}{c}17 / 31 \\
(55 \%)\end{array}$ & $\begin{array}{l}17 / 29 \\
(59 \%)\end{array}$ & $\begin{array}{l}16 / 18 \\
(89 \%)\end{array}$ & $\begin{array}{l}15 / 19 \\
(79 \%)\end{array}$ & $\begin{array}{l}1 / 2 \\
(50 \%)\end{array}$ & $\begin{array}{c}2 / 5 \\
(40 \%)\end{array}$ & $\begin{array}{c}2 / 2 \\
(100 \%)\end{array}$ & $\begin{array}{r}1 / 2 \\
(50 \%)\end{array}$ \\
\hline $\begin{array}{l}\text { Relapse or } \\
\text { breakthrough }\end{array}$ & $\begin{array}{r}4 / 24 \\
(17 \%)\end{array}$ & $\begin{array}{l}10 / 23 \\
(44 \%)\end{array}$ & $\begin{array}{r}2 / 12 \\
(17 \%)\end{array}$ & $\begin{array}{l}7 / 18 \\
(39 \%)\end{array}$ & $\begin{array}{l}13 / 41 \\
(32 \%)\end{array}$ & $\begin{array}{l}19 / 35 \\
(54 \%)\end{array}$ & $\begin{array}{l}14 / 31 \\
(45 \%)\end{array}$ & $\begin{array}{l}12 / 29 \\
(41 \%)\end{array}$ & $\begin{array}{r}2 / 18 \\
(11 \%)\end{array}$ & $\begin{array}{r}4 / 19 \\
(21 \%)\end{array}$ & $\begin{array}{c}1 / 2 \\
(50 \%)\end{array}$ & $\begin{array}{l}3 / 5 \\
(60 \%)\end{array}$ & 0 & $\begin{array}{r}1 / 2 \\
(50 \%)\end{array}$ \\
\hline $\begin{array}{l}\text { Years since } \\
\text { transplantation }\end{array}$ & $\cdot$ &. &.$\cdot$ & $\cdot$ & $\begin{array}{c}3 \cdot 3 \\
(1 \cdot 9-5 \cdot 5)\end{array}$ & $\begin{array}{c}2 \cdot 7 \\
(1 \cdot 6-5 \cdot 8)\end{array}$ & $\begin{array}{c}4 \cdot 4 \\
(2 \cdot 4-9 \cdot 9)\end{array}$ & $\begin{array}{c}6 \cdot 7 \\
(4 \cdot 1-9 \cdot 2)\end{array}$ & $\begin{array}{l}8 \cdot 7(4 \cdot 1- \\
12 \cdot 0)\end{array}$ & $\begin{array}{l}2 \cdot 5 \\
(1 \cdot 0-9 \cdot 1)\end{array}$ & $\begin{array}{l}6 \cdot 0 \\
(3 \cdot 2-7 \cdot 2)\end{array}$ & $\begin{array}{l}9 \cdot 5 \\
(7 \cdot 9-9 \cdot 5)\end{array}$ & $\begin{array}{c}0.4 \\
(0 \cdot 4-1 \cdot 5)\end{array}$ & $\begin{array}{c}0 \cdot 3 \\
(0 \cdot 2-0 \cdot 4)\end{array}$ \\
\hline eGFR (mL/min) & $\begin{array}{l}90 \\
(70-117)\end{array}$ & $\begin{array}{l}92 \\
(71-113)\end{array}$ & $\begin{array}{l}77 \\
(67-90)\end{array}$ & $\begin{array}{l}96 \\
(71-112)\end{array}$ & $\begin{array}{c}61 \\
(55-73)\end{array}$ & $\begin{array}{l}66 \\
(52-76)\end{array}$ & $\begin{array}{l}66 \\
(51-79)\end{array}$ & $\begin{array}{c}61 \\
(51-74)\end{array}$ & $\begin{array}{l}76 \\
(54-90)\end{array}$ & $\begin{array}{l}61 \\
(51-73)\end{array}$ & $\begin{array}{l}97 \\
(60-100)\end{array}$ & $\begin{array}{l}51 \\
(51-74)\end{array}$ & $\begin{array}{l}88 \\
(80-94)\end{array}$ & $\begin{array}{l}89 \\
(75-103)\end{array}$ \\
\hline $\begin{array}{l}\text { Platelets } \\
\left(\times 10^{3} \text { per } \mu \mathrm{L}\right)\end{array}$ & $\begin{array}{l}75 \\
(57-89)\end{array}$ & $\begin{array}{l}85 \\
(55-126)\end{array}$ & $\begin{array}{l}73 \\
(57-111)\end{array}$ & $\begin{array}{l}74 \\
(60-89)\end{array}$ & $\begin{array}{l}166 \\
(131,204)\end{array}$ & $\begin{array}{l}151 \\
(120-191)\end{array}$ & $\begin{array}{l}101 \\
(84-145)\end{array}$ & $\begin{array}{l}108 \\
(88-138)\end{array}$ & $\begin{array}{l}97 \\
(72-131)\end{array}$ & $\begin{array}{l}90 \\
(68-128)\end{array}$ & $\begin{array}{l}75 \\
(52-75)\end{array}$ & $\begin{array}{l}60 \\
(47-88)\end{array}$ & $\begin{array}{l}173 \\
(149-174)\end{array}$ & $\begin{array}{l}171 \\
(158-183)\end{array}$ \\
\hline \multicolumn{15}{|l|}{$\begin{array}{l}\text { Child-Turcotte- } \\
\text { Pugh class }\end{array}$} \\
\hline Class A (5-6) & $\begin{array}{c}1 \\
(4 \%)\end{array}$ & $\begin{array}{c}1 \\
(4 \%)\end{array}$ & 0 & 0 & .. & .. & $\begin{array}{c}33 \\
(97 \%)\end{array}$ & $\begin{array}{c}33 \\
(100 \%)\end{array}$ & $\begin{array}{l}3 \\
(14 \%)\end{array}$ & $\begin{array}{c}2 \\
(9 \%)\end{array}$ & 0 & 0 & .. &. \\
\hline Class B (7-9) & $\begin{array}{l}26 \\
(93 \%)\end{array}$ & $\begin{array}{l}26 \\
(93 \%)\end{array}$ & $\begin{array}{l}7 \\
(28 \%)\end{array}$ & $\begin{array}{c}1 \\
(4 \%)\end{array}$ & .. & .* & $\begin{array}{r}1 \\
(3 \%)\end{array}$ & 0 & $\begin{array}{l}19 \\
(86 \%)\end{array}$ & $\begin{array}{l}19 \\
(83 \%)\end{array}$ & 0 & $\begin{array}{c}1 \\
(20 \%)\end{array}$ & .. & .. \\
\hline \multirow[t]{2}{*}{ Class C (10-12) } & $\begin{array}{c}1 \\
(4 \%)\end{array}$ & $\begin{array}{c}1 \\
(4 \%)\end{array}$ & $\begin{array}{l}18 \\
(72 \%)\end{array}$ & $\begin{array}{l}25 \\
(96 \%)\end{array}$ &. & .. & 0 & 0 & 0 & $\begin{array}{l}2 \\
(9 \%)\end{array}$ & $\begin{array}{l}3 \\
(100 \%)\end{array}$ & $\begin{array}{l}4 \\
(80 \%)\end{array}$ & .. &.. \\
\hline & & & & & & & & & & & & \multicolumn{3}{|c|}{ (Table 1 continues on next page } \\
\hline
\end{tabular}




\begin{tabular}{|c|c|c|c|c|c|c|c|c|c|c|c|c|c|c|}
\hline & \multicolumn{4}{|c|}{ Cohort A: before transplantation } & \multicolumn{10}{|c|}{ Cohort B: after transplantation } \\
\hline & \multicolumn{2}{|c|}{ Group 1, CTP-B } & \multicolumn{2}{|c|}{ Group 2, CTP-C } & \multicolumn{2}{|c|}{ Group 3, no cirrhosis } & \multicolumn{2}{|c|}{ Group 4, CTP-A } & \multicolumn{2}{|c|}{ Group 5, CTP-B } & \multicolumn{2}{|c|}{ Group 6, CTP-C } & \multicolumn{2}{|c|}{ Group 7, FCH } \\
\hline & $\begin{array}{l}12 \text { weeks } \\
(n=28)\end{array}$ & $\begin{array}{l}24 \text { weeks } \\
(n=28)\end{array}$ & $\begin{array}{l}12 \text { weeks } \\
(n=25)\end{array}$ & $\begin{array}{l}24 \text { weeks } \\
(n=26)\end{array}$ & $\begin{array}{l}12 \text { weeks } \\
(n=52)\end{array}$ & $\begin{array}{l}24 \text { weeks } \\
(n=49)\end{array}$ & $\begin{array}{l}12 \text { weeks } \\
(\mathrm{n}=34)\end{array}$ & $\begin{array}{l}24 \text { weeks } \\
(n=33)\end{array}$ & $\begin{array}{l}12 \text { weeks } \\
(n=22)\end{array}$ & $\begin{array}{l}24 \text { weeks } \\
(n=23)\end{array}$ & $\begin{array}{l}12 \text { weeks } \\
(n=3)\end{array}$ & $\begin{array}{l}24 \text { weeks } \\
(n=5)\end{array}$ & $\begin{array}{l}12 \text { weeks } \\
(n=3)\end{array}$ & $\begin{array}{l}24 \text { weeks } \\
(n=2)\end{array}$ \\
\hline \multicolumn{15}{|c|}{ (Continued from previous page) } \\
\hline \multicolumn{15}{|c|}{ MELD score } \\
\hline$<10$ & $\begin{array}{c}2 \\
(7 \%)\end{array}$ & $\begin{array}{l}6 \\
(21 \%)\end{array}$ & $\begin{array}{c}2 \\
(8 \%)\end{array}$ & 0 &. &. & $\begin{array}{c}19 \\
(56 \%)\end{array}$ & $\begin{array}{c}17 \\
(52 \%)\end{array}$ & $\begin{array}{c}5 \\
(23 \%)\end{array}$ & $\begin{array}{l}7 \\
(30 \%)\end{array}$ & 0 & 0 &. &. \\
\hline $10-15$ & $\begin{array}{l}22 \\
(79 \%)\end{array}$ & $\begin{array}{l}18 \\
(64 \%)\end{array}$ & $\begin{array}{l}11 \\
(44 \%)\end{array}$ & $\begin{array}{l}15 \\
(58 \%)\end{array}$ & .. & .. & $\begin{array}{c}15 \\
(44 \%)\end{array}$ & $\begin{array}{c}16 \\
(48 \%)\end{array}$ & $\begin{array}{l}13 \\
(59 \%)\end{array}$ & $\begin{array}{l}13 \\
(57 \%)\end{array}$ & $\begin{array}{c}1 \\
(33 \%)\end{array}$ & $\begin{array}{l}4 \\
(80 \%)\end{array}$ & .. & .. \\
\hline $16-20$ & $\begin{array}{l}4 \\
(14 \%)\end{array}$ & $\begin{array}{l}4 \\
(14 \%)\end{array}$ & $\begin{array}{l}10 \\
(40 \%)\end{array}$ & $\begin{array}{c}9 \\
(35 \%)\end{array}$ & .. &. & 0 & $\begin{array}{c}2 \\
(8 \%)\end{array}$ & $\begin{array}{l}4 \\
(18 \%)\end{array}$ & $\begin{array}{c}3 \\
(13 \%)\end{array}$ & $\begin{array}{l}2 \\
(67 \%)\end{array}$ & $\begin{array}{l}1 \\
(20 \%)\end{array}$ & .. &. \\
\hline $21-25$ & 0 & 0 & $\begin{array}{l}2 \\
(8 \%)\end{array}$ & $\begin{array}{c}2 \\
(8 \%)\end{array}$ & .. &. & 0 & 0 & 0 & 0 & 0 & 0 & .. &. \\
\hline $\begin{array}{l}\text { Data are } n \\
\text { estimated } \\
\text { Pl=proteas } \\
(n=3) \text {, ribav }\end{array}$ & $\begin{array}{l}\mathrm{PEG}=\text { pegint } \\
\text { herapy }(\mathrm{n}=\end{array}$ & $\begin{array}{l}\text { R), or mean } \\
\text { oft-Gault Eq } \\
\text { erferon-alph } \\
\text { ), and unknc }\end{array}$ & $\begin{array}{l}\text { *Other pre } \\
\text { wn }(n=1) \text {. }\end{array}$ & $\begin{array}{l}\text { therwise stat } \\
\text { esser of actua } \\
\text { vious regimen }\end{array}$ & nclude int & eron monc & rapy $(n=6), i$ & nterferon $\mathrm{pl}$ & bavirin $(n=6$ & 6), peginterfe & $\begin{array}{l}\text { MELD=Mode } \\
\text { ron monothe }\end{array}$ & $\begin{array}{l}\text { isits. Glom } \\
\text { el for End-st } \\
\text { erapy }(n=6)\end{array}$ & $\begin{array}{l}\text { lar filtration } \\
\text { e Liver Disea } \\
\text { ofosbuvir pl }\end{array}$ & $\begin{array}{l}\text { ate } \\
\text { e. } \\
\text { ribavirin }\end{array}$ \\
\hline
\end{tabular}

\begin{tabular}{|c|c|c|c|c|c|c|c|c|c|c|c|c|c|c|}
\hline & \multicolumn{4}{|c|}{ Cohort A: before transplantation } & \multicolumn{10}{|c|}{ Cohort B: after transplantation } \\
\hline & \multicolumn{2}{|c|}{ Group 1, CTP-B } & \multicolumn{2}{|c|}{ Group 2, CTP-C } & \multicolumn{2}{|c|}{ Group 3, no cirrhosis } & \multicolumn{2}{|c|}{ Group 4, CTP-A } & \multicolumn{2}{|c|}{ Group 5, CTP-B } & \multicolumn{2}{|c|}{ Group 6, CTP-C } & \multicolumn{2}{|c|}{ Group 7, FCH } \\
\hline & $\begin{array}{l}12 \text { weeks } \\
(n=26)\end{array}$ & $\begin{array}{l}24 \text { weeks } \\
(n=27)\end{array}$ & $\begin{array}{l}12 \text { weeks } \\
(n=25)\end{array}$ & $\begin{array}{l}24 \text { weeks } \\
(n=26)\end{array}$ & $\begin{array}{l}12 \text { weeks } \\
(n=52)\end{array}$ & $\begin{array}{l}24 \text { weeks } \\
(n=49)\end{array}$ & $\begin{array}{l}12 \text { weeks } \\
(n=34)\end{array}$ & $\begin{array}{l}24 \text { weeks } \\
(n=33)\end{array}$ & $\begin{array}{l}12 \text { weeks } \\
(n=22)\end{array}$ & $\begin{array}{l}24 \text { weeks } \\
(n=23)\end{array}$ & $\begin{array}{l}12 \text { weeks } \\
(n=3)\end{array}$ & $\begin{array}{l}24 \text { weeks } \\
(n=5)\end{array}$ & $\begin{array}{l}12 \text { weeks } \\
(n=3)\end{array}$ & $\begin{array}{l}24 \text { weeks } \\
(\mathrm{n}=2)\end{array}$ \\
\hline \multicolumn{15}{|c|}{ HCV genotype 1} \\
\hline SVR12 & $\begin{array}{l}20 / 23 \\
(87 \% \\
70-96)\end{array}$ & $\begin{array}{l}22 / 23 \\
(96 \%, \\
81-100)\end{array}$ & $\begin{array}{l}17 / 20 \\
(85 \% \\
66-96)\end{array}$ & $\begin{array}{l}18 / 23 \\
(78 \%, \\
60-91)\end{array}$ & $\begin{array}{l}42 / 45 \\
(93 \% \\
84-98)\end{array}$ & $\begin{array}{l}44 / 44 \\
(100 \% \\
93-100)\end{array}$ & $\begin{array}{l}30 / 30 \\
(100 \% \\
91-100)\end{array}$ & $\begin{array}{l}27 / 28 \\
(96 \% \\
84-100)\end{array}$ & $\begin{array}{l}19 / 20 \\
(95 \%, \\
78-100)\end{array}$ & $\begin{array}{l}20 / 20 \\
(100 \% \\
86-100)\end{array}$ & $\begin{array}{l}1 / 2 \\
(50 \% \\
3-98)\end{array}$ & $\begin{array}{l}4 / 5 \\
(80 \% \\
34-99)\end{array}$ & $\begin{array}{l}3 / 3 \\
(100 \% \\
37-100)\end{array}$ & $\begin{array}{l}2 / 2 \\
(100 \% \\
22-100)\end{array}$ \\
\hline Relapse & 3 & 1 & 1 & 1 & 1 & 0 & 0 & 0 & 0 & 0 & 0 & 0 & 0 & 0 \\
\hline Death & 0 & 0 & 2 & 3 & 2 & 0 & 0 & 1 & 1 & 0 & 1 & 1 & 0 & 0 \\
\hline Other & 0 & 0 & 0 & 1 & 0 & 0 & 0 & 0 & 0 & 0 & 0 & 0 & 0 & 0 \\
\hline \multicolumn{15}{|c|}{ HCV genotype 4} \\
\hline SVR12 & $\begin{array}{c}2 / 3 \\
(67 \% \\
14-98)\end{array}$ & $\begin{array}{c}2 / 2 \\
(100 \% \\
22-100)\end{array}$ & $\begin{array}{c}0 / 1 \\
(0 \% \\
0-95)\end{array}$ & $\begin{array}{c}1 / 2 \\
(50 \% \\
3-98)\end{array}$ & $\begin{array}{c}7 / 7 \\
(100 \% \\
65-100)\end{array}$ & $\begin{array}{c}5 / 5 \\
(100 \% \\
55-100)\end{array}$ & $\begin{array}{c}3 / 4 \\
(75 \% \\
25-99)\end{array}$ & $\begin{array}{c}5 / 5 \\
(100 \% \\
55-100)\end{array}$ & $\begin{array}{c}2 / 2 \\
(100 \% \\
22-100)\end{array}$ & $\begin{array}{c}3 / 3 \\
(100 \% \\
37-100)\end{array}$ & $\begin{array}{l}0 / 1 \\
(0 \%, \\
0-95)\end{array}$ & .. & .. & .. \\
\hline Relapse & 1 & 0 & 1 & 0 & 0 & 0 & 0 & 0 & 0 & 0 & 1 & .. & .. & .. \\
\hline Death & 0 & 0 & 0 & 1 & 0 & 0 & 1 & 0 & 0 & 0 & 0 &.. & .. & .. \\
\hline Other & 0 & 0 & 0 & 0 & 0 & 0 & 0 & 0 & 0 & 0 & 0 & .. & .. & .. \\
\hline
\end{tabular}

treatment and 16 (94\%, 75-100) of 17 patients receiving 24 weeks of treatment. Of the five patients who did not achieve SVR12, three-all receiving 12 weeks of treatment-had virological relapse, and two died (one post-transplantation CTP-A on 12 weeks of treatment, and one untransplanted CTP-C on 24 weeks of treatment). All three patients who had virological relapse were patients with decompensated disease (one CTP-B and one CTP-C who had not received a previous liver transplant, one CTP-C who had received a liver transplant), who were treated for 12 weeks, and had a baseline NS5A resistance-associated variant. No patient with genotype $4 \mathrm{HCV}$ who received 24 weeks of treatment had virological relapse. Two patients with genotype 4 HCV were not included in the SVR12 analysis: one cohort A patient was found to have a CTP score of 5 upon recalculation of screening score (required to be $\geq 7$ to enrol) and therefore did not meet the eligibility criteria for study and one received a liver transplant before posttreatment week 12. This patient had HCV RNA less than LLOQ at the time of transplantation and continued to have HCV RNA less than LLOQ 12 weeks after transplantation.

During the study, ten patients (nine in cohort A and one in cohort B) underwent liver transplantation: four during the treatment phase, one after post-treatment 
week 2 , two after post-treatment week 8 , and three after post-treatment week 12. Six patients had CTP-C disease, and four had CTP-B disease. All received at least 11 weeks of study treatment before undergoing transplantation. HCV RNA was undetectable in all ten patients at the time of transplantation, and none developed recurrent $\mathrm{HCV}$ infection. The patient in cohort B underwent retransplantation after achieving SVR12. Of the ten patients, all achieved pTVR12.

NS5A pretreatment resistance analysis was done for 308 patients: 276 with genotype 1 infection and 32 with genotype 4 infection. NS5B pretreatment resistance

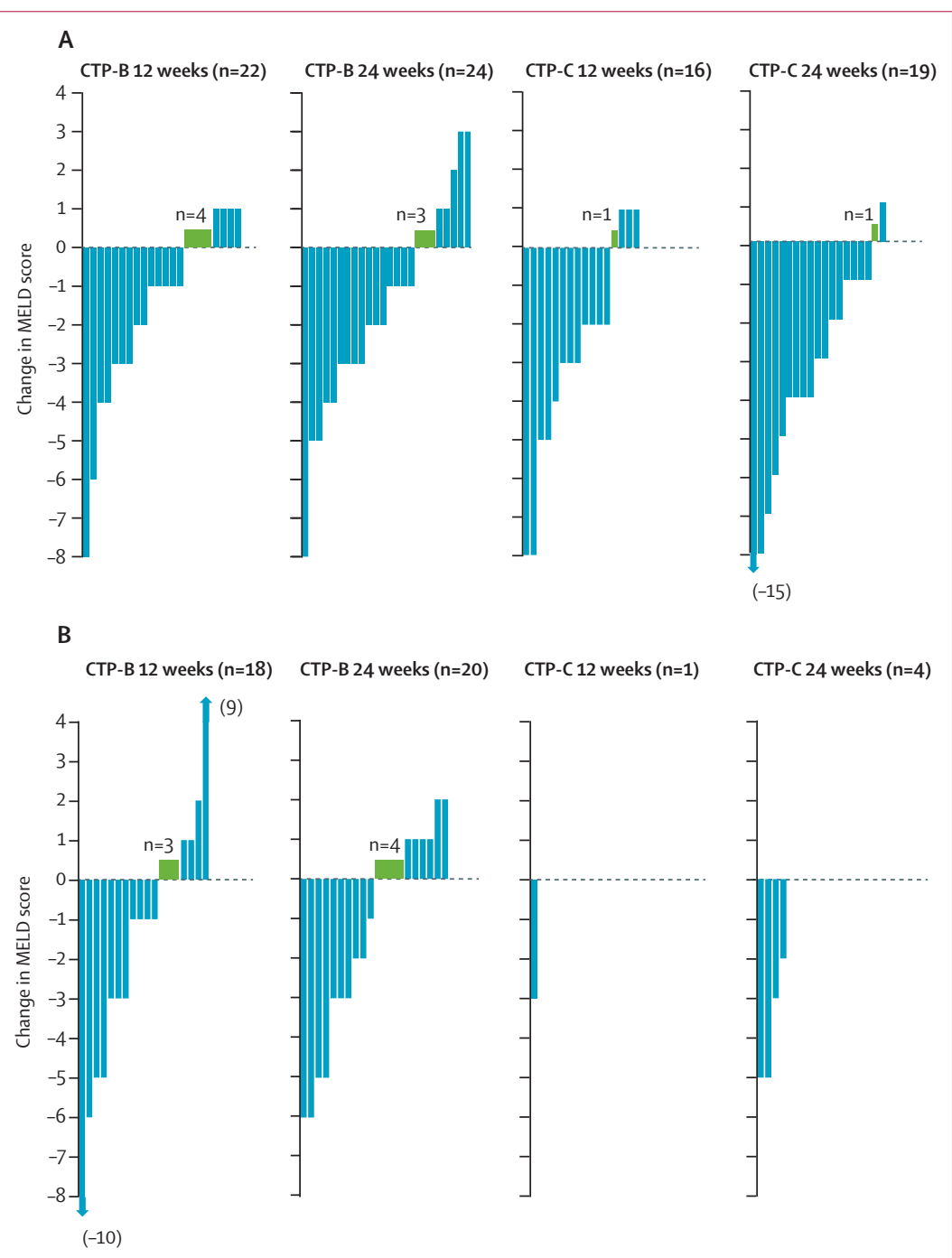

Figure 2: Individual Model for End-Stage Liver Disease (MELD) score changes from baseline to post-treatment week 12 in pre-transplantation and post-transplantation decompensated (Child-Turcotte-Pugh [CTP]-B or (TP-C) patients who achieved sustained virological response 12 weeks after treatment

Change from baseline in MELD scores in non-transplanted patients in groups 1 and 2 (A) and post-transplantation patients in groups 5 and 6 (B). MELD score $=10 \times\left(\left[0.957 \times \log _{e}(\mathrm{Scr})\right]+\left[0.378 \times \log _{e}(\right.\right.$ Tbil $\left.)\right]+\left[1.12 \times \log _{e}(\right.$ INR $\left.\left.)\right]+0.643\right)$ where $\mathrm{Scr}$ is serum creatinine $(\mathrm{mg} / \mathrm{dL})$, Thil is total bilirubin $(\mathrm{mg} / \mathrm{dL})$, and INR is international normalisation ratio. Laboratory values less than 1.0 were set to 1 in formula; for patients receiving dialysis at least two times in the last week, serum creatinine was set to $4.0 \mathrm{mg} / \mathrm{dL}$ for the calculation. Missing follow-up at post-treatment week 12 (A) CTP-C, 12 weeks ( $n=1)$; (B) CTP-B, 12 weeks ( $n=3)$, CTP-B, 24 weeks $(n=3)$. analysis was done for 305 patients: 275 with genotype 1 and 30 with genotype 4 infection. Using a $1 \%$ deep sequencing cutoff, $59(21 \%)$ of 276 patients with genotype $1 \mathrm{HCV}$ infection had baseline NS5A resistanceassociated variants that confer reduced susceptibility to ledipasvir. Of these 59 patients, only two (3\%) relapsed after 12 weeks of treatment. Five $(2 \%)$ of 217 patients without baseline NS5A resistance-associated variants relapsed, three after 12 weeks of treatment and two after 24 weeks of treatment. None of the 32 patients with NS5A resistance-associated variants who received ledipasvir-sofosbuvir plus ribavirin for 24 weeks relapsed. Of the 32 patients with genotype $4 \mathrm{HCV}$ infection, 24 (75\%) had NS5A resistance-associated variants. Three of them had virological relapse. Of the eight genotype 4 patients without baseline NS5A mutations, none had virological relapse.

Overall, ten patients had virological relapse: seven with genotype 1 infection (five with genotype 1a and two with genotype $1 \mathrm{~b}$ ) and three with genotype 4 infection. Nine of the ten patients who relapsed had decompensated cirrhosis. Two (29\%) of seven patients with genotype 1 HCV infection who relapsed had NS5A resistanceassociated variants at pretreatment (positions Q30, L31, and Y93). At virological failure, all patients had NS5A variants: K24R, M28T, Q30H/K/R, L31M/V, and Y93H/C. The three patients with genotype $4 \mathrm{HCV}$ who relapsed had $\mathrm{L} 30 \mathrm{H} / \mathrm{R}, \mathrm{M} 31 \mathrm{~V}, \mathrm{P} 58 \mathrm{~L}$, and Y93C at the time of relapse.

At the time of relapse, the nucleotide inhibitor resistance-associated variant S282T was not recorded in any of the patients. Two patients with genotype 1a and genotype 4d infection had the variant E237G at the time of relapse. The clinical significance of E237G is currently unknown.

We assessed the relation between achieving SVR12 and changes in MELD and CTP scores (figures 2 and 3). Improvements in MELD scores occurred in 58 (72\%) of 81 non-transplanted patients who achieved SVR12 and had measurements for MELD recorded for both baseline and week 12 of follow-up (figure 2A). A MELD score of at least 15 is generally the threshold at which liver transplantation is recommended..$^{12}$ Although change in transplant eligibility was not specifically captured in the study, among non-transplanted patients whose MELD score was at least 15 at baseline, $20(80 \%)$ of 25 patients had MELD scores less than 15 at post-treatment week 12 . Conversely, among non-transplanted patients whose MELD score was less than 15 at baseline, two (4\%) of 56 had a MELD score of at least 15 at post-treatment week 12 . For this same group of patients, 14 (28\%) of 50 improved from CTP-B at baseline to CTP-A at post-treatment week 12 and 21 (68\%) of 31 improved from CTP-C at baseline to CTP-B cirrhosis at post-treatment week 12 .

Most patients with either CTP-B or CTP-C disease who had undergone liver transplantation also had improved MELD scores (25/43, 58\%). Among post-transplanted patients with decompensated cirrhosis whose MELD 
score was at least 15 at baseline, five $(63 \%)$ of eight had MELD scores less than 15 at post-treatment week 12 . Conversely, among those whose MELD score was less than 15 at baseline, three (9\%) of 35 had a MELD score of at least 15 at post-treatment week 12 . For this same group of patients, 17 (52\%) of 33 improved from CTP-B at baseline to CTP-A at post-treatment week 12, and three $(60 \%)$ of five improved from CTP-C to CTP-B cirrhosis.

Rates of adverse events were consistent with the study population having advanced liver disease (table 3). However, 316 (95\%) of 333 patients completed study treatment, and only seven patients $(2 \%)$ discontinued ledipasvir-sofosbuvir dosing prematurely because of an adverse event (table 3). Of the adverse events leading to discontinuation from ledipasvir-sofosbuvir, only one event, hepatocellular carcinoma, led to discontinuation in more than one patient $(n=2) .72$ patients $(22 \%)$ had serious adverse events (appendix). Nine serious adverse events were considered treatment-related: anaemia $(n=5)$, diarrhoea $(n=1)$, fall $(n=1)$, hyperbilirubinaemia $(\mathrm{n}=1)$, and vomiting $(\mathrm{n}=1)$. The fall was not considered related to ledipasvir-sofosbuvir by the investigator, but rather due to ribavirin-mediated anaemia.

Among the 226 post-transplantation patients in cohort B included in the efficacy analysis, the most commonly used immunosuppressants were tacrolimus or tacrolimus monohydrate in 144 patients (64\%), mycophenolate mofetil, mycophenolate sodium, or mycophenolic acid in 100 patients (44\%), and cyclosporine in 63 patients (28\%). Less commonly used immunosuppressants were sirolimus (11 patients [5\%]) and everolimus (nine patients [4\%]). No patients had changes in immunosuppressant medications that were attributed to drug interaction with study treatment. Ten patients required an increase in immunosuppressant dosing (tacrolimus for all ten), possibly because of altered hepatic function after viral clearance.

17 patients died during the study (appendix). Of these, ten patients died within 30 days of treatment; seven patients died more than 30 days after the end of treatment. 11 (65\%) of the 17 deaths occurred in patients with decompensated CTP-C cirrhosis. Additionally, two patients with decompensated CTP-B cirrhosis died; one because of a decompensation event and the other because of small bowel pneumatosis. Two patients with stage F0-F3 fibrosis died from bronchial carcinoma and graft failure secondary to rejection, respectively. Two patients with compensated (CTP-A) cirrhosis died, one from myocardial infarction and the other from hepatic decompensation.

Across all groups, the most common grade 3 or 4 laboratory abnormalities were decreases in haemoglobin and lymphocytes and increases in total bilirubin and glucose. Median creatinine concentrations in all groups remained stable while on treatment. Decreases in haemoglobin and lymphocytes are known complications of ribavirin therapy, which in this study probably exacerbated pre-existing anaemia and lymphopenia.

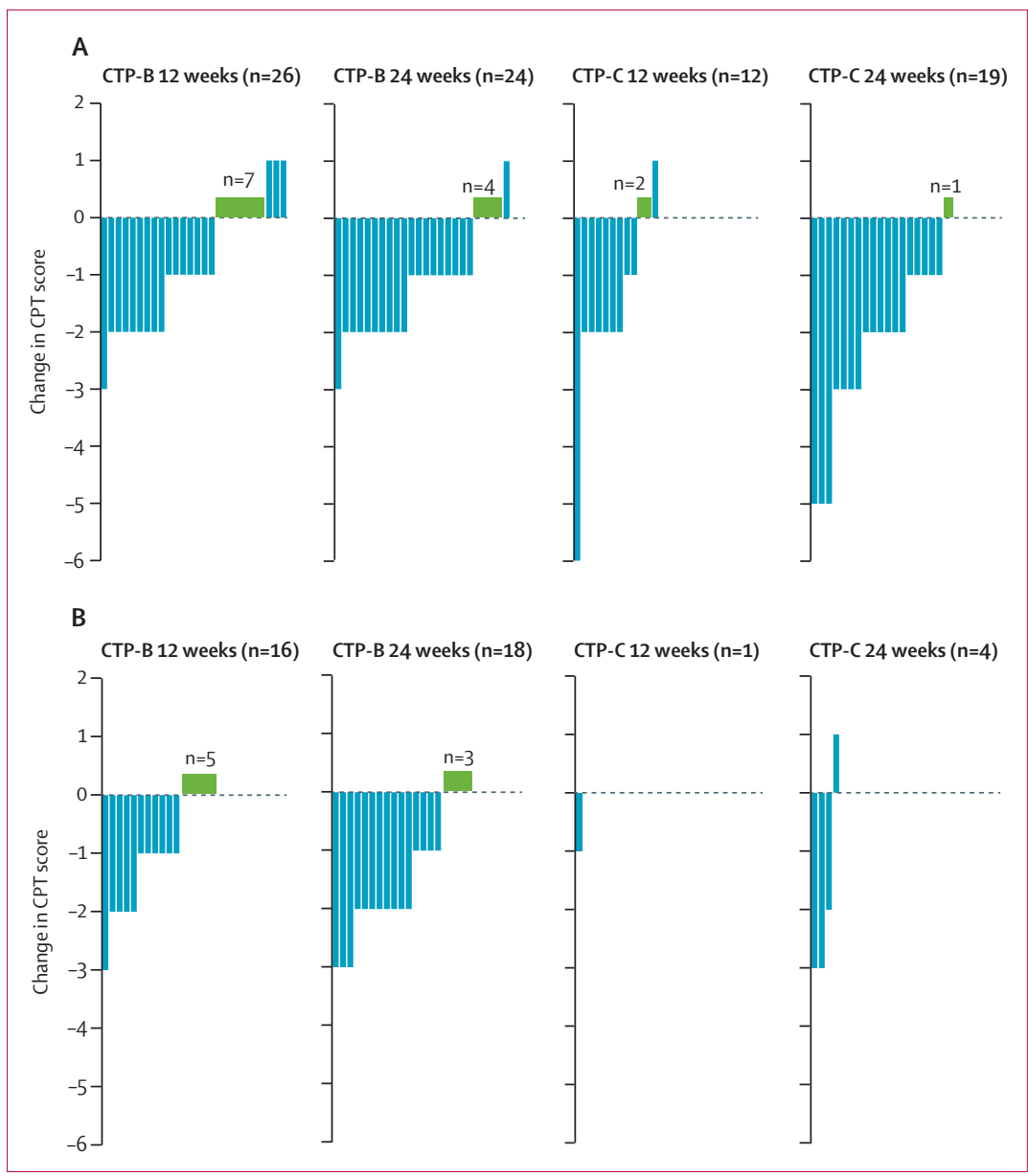

Figure 3: Individual changes in Child-Turcotte-Pugh (CTP) scores from baseline to post-treatment week 12 in pre-transplantation and post-transplantation decompensated (CTP-B or CTP-C) patients who achieved sustained virological response 12 weeks after treatment

Change from baseline in CTP scores in non-transplanted patients in groups 1 and 2 (A) and post-transplantation patients in groups 5 and 6 (B). CTP score was calculated as the sum of the five scores assigned for total bilirubin, serum albumin, international normalised ratio, ascites, and hepatic encephalopathy. Missing follow-up at post-treatment week 12: (A) CTP-C, 12 weeks ( $n=1)$; (B) CTP-B, 12 weeks ( $n=3)$, CTP-B, 24 weeks ( $n=2)$, CTP-C, 24 weeks $(n=1)$

Increases in total bilirubin also occurred, almost exclusively due to an increase in indirect bilirubin, which is another known complication of ribavirin therapy. Glucose abnormalities were only recorded in patients with known pre-existing issues with glycaemic control.

The average daily dose of ribavirin in patients with decompensated cirrhosis (non-transplanted or posttransplantation) was about $600 \mathrm{mg}$. The average daily dose of ribavirin in patients with no cirrhosis or compensated cirrhosis was about $800 \mathrm{mg}$. Ribavirin dosing was reduced in 134 patients (40\%), interrupted in 26 patients $(8 \%)$, and discontinued in 40 patients $(12 \%)$. Dose discontinuation was not associated with virological relapse.

\section{Discussion}

In this open-label trial, the all-oral combination of ledipasvir-sofosbuvir plus ribavirin for 12 or 24 weeks 


\begin{tabular}{|c|c|c|c|c|c|c|c|c|c|c|c|c|c|c|}
\hline & \multicolumn{4}{|c|}{ Cohort A: before transplantation } & \multicolumn{10}{|c|}{ Cohort B: after transplantation } \\
\hline & \multicolumn{2}{|l|}{ CTP-B } & \multicolumn{2}{|l|}{ CTP-C } & \multicolumn{2}{|c|}{ No cirrhosis } & \multicolumn{2}{|l|}{ CTP-A } & \multicolumn{2}{|l|}{ CTP-B } & \multicolumn{2}{|l|}{ CTP-C } & \multicolumn{2}{|l|}{$\mathrm{FCH}$} \\
\hline & $\begin{array}{l}12 \text { weeks } \\
(n=28)\end{array}$ & $\begin{array}{l}24 \text { weeks } \\
(n=28)\end{array}$ & $\begin{array}{l}12 \text { weeks } \\
(\mathrm{n}=25)\end{array}$ & $\begin{array}{l}24 \text { weeks } \\
(n=26)\end{array}$ & $\begin{array}{l}12 \text { weeks } \\
(n=52)\end{array}$ & $\begin{array}{l}24 \text { weeks } \\
(n=49)\end{array}$ & $\begin{array}{l}12 \text { weeks } \\
(n=34)\end{array}$ & $\begin{array}{l}24 \text { weeks } \\
(n=33)\end{array}$ & $\begin{array}{l}12 \text { weeks } \\
(n=22)\end{array}$ & $\begin{array}{l}24 \text { weeks } \\
(n=23)\end{array}$ & $\begin{array}{l}12 \text { weeks } \\
(n=3)\end{array}$ & $\begin{array}{l}24 \text { weeks } \\
(n=5)\end{array}$ & $\begin{array}{l}12 \text { weeks } \\
(n=3)\end{array}$ & $\begin{array}{l}24 \text { weeks } \\
(n=2)\end{array}$ \\
\hline Any adverse event & $\begin{array}{l}27 \\
(96 \%)\end{array}$ & $\begin{array}{l}27 \\
(96 \%)\end{array}$ & $\begin{array}{l}24 \\
(96 \%)\end{array}$ & $\begin{array}{l}25 \\
(96 \%)\end{array}$ & $\begin{array}{l}51 \\
(98 \%)\end{array}$ & $\begin{array}{l}48 \\
(98 \%)\end{array}$ & $\begin{array}{l}28 \\
(82 \%)\end{array}$ & $\begin{array}{l}31 \\
(94 \%)\end{array}$ & $\begin{array}{l}21 \\
(95 \%)\end{array}$ & $\begin{array}{l}20 \\
(87 \%)\end{array}$ & $\begin{array}{l}3 \\
(100 \%)\end{array}$ & $\begin{array}{l}5 \\
(100 \%)\end{array}$ & $\begin{array}{l}3 \\
(100 \%)\end{array}$ & $\begin{array}{l}2 \\
(100 \%)\end{array}$ \\
\hline Serious adverse events & $\begin{array}{c}3 \\
(11 \%)\end{array}$ & $\begin{array}{l}6 \\
(21 \%)\end{array}$ & $\begin{array}{l}13 \\
(52 \%)\end{array}$ & $\begin{array}{c}9 \\
(35 \%)\end{array}$ & $\begin{array}{c}9 \\
(17 \%)\end{array}$ & $\begin{array}{l}5 \\
(10 \%)\end{array}$ & $\begin{array}{l}3 \\
(9 \%)\end{array}$ & $\begin{array}{l}7 \\
(21 \%)\end{array}$ & $\begin{array}{l}5 \\
(23 \%)\end{array}$ & $\begin{array}{l}6 \\
(26 \%)\end{array}$ & $\begin{array}{l}1 \\
(33 \%)\end{array}$ & $\begin{array}{l}2 \\
(40 \%)\end{array}$ & $\begin{array}{l}2 \\
(67 \%)\end{array}$ & $\begin{array}{l}1 \\
(50 \%)\end{array}$ \\
\hline Deaths* & 0 & 0 & $\begin{array}{c}1 \\
(4 \%)\end{array}$ & $\begin{array}{l}3 \\
(12 \%)\end{array}$ & $\begin{array}{c}1 \\
(2 \%)\end{array}$ & 0 & $\begin{array}{c}1 \\
(3 \%)\end{array}$ & 0 & $\begin{array}{c}1 \\
(5 \%)\end{array}$ & 0 & $\begin{array}{l}1 \\
(33 \%)\end{array}$ & $\begin{array}{l}1 \\
(20 \%)\end{array}$ & 0 & 0 \\
\hline \multicolumn{15}{|c|}{ Adverse events leading to discontinuation } \\
\hline \multicolumn{15}{|l|}{ Ledipasvir-sofosbuvir } \\
\hline Oedema & 0 & 0 & 0 & $\begin{array}{c}1 \\
(4 \%)\end{array}$ & 0 & 0 & 0 & 0 & 0 & 0 & 0 & 0 & 0 & 0 \\
\hline Dehydration & 0 & 0 & 0 & 0 & 0 & 0 & 0 & 0 & 0 & 0 & 0 & $\begin{array}{l}1 \\
(20 \%)\end{array}$ & 0 & 0 \\
\hline Diabetes type 2 & 0 & 0 & 0 & 0 & 0 & 0 & 0 & $\begin{array}{c}1 \\
(3 \%)\end{array}$ & 0 & 0 & 0 & 0 & 0 & 0 \\
\hline $\begin{array}{l}\text { Hepatocellular } \\
\text { carcinoma }\end{array}$ & $\begin{array}{c}1 \\
(4 \%)\end{array}$ & 0 & 0 & $\begin{array}{c}1 \\
(4 \%)\end{array}$ & 0 & 0 & 0 & 0 & 0 & 0 & 0 & 0 & 0 & 0 \\
\hline Hepatic failure & 0 & 0 & 0 & 0 & $\begin{array}{c}1 \\
(2 \%)\end{array}$ & 0 & 0 & 0 & 0 & 0 & 0 & 0 & 0 & 0 \\
\hline Hyperbilirubinaemia & 0 & $\begin{array}{c}1 \\
(4 \%)\end{array}$ & 0 & 0 & 0 & 0 & 0 & 0 & 0 & 0 & 0 & 0 & 0 & 0 \\
\hline \multicolumn{15}{|l|}{ Ribavirin } \\
\hline Anaemia & 0 & $\begin{array}{c}1 \\
(4 \%)\end{array}$ & $\begin{array}{l}3 \\
(12 \%)\end{array}$ & $\begin{array}{l}3 \\
(12 \%)\end{array}$ & $\begin{array}{c}2 \\
(4 \%)\end{array}$ & $\begin{array}{l}1 \\
(2 \%)\end{array}$ & 0 & $\begin{array}{l}3 \\
(9 \%)\end{array}$ & $\begin{array}{l}2 \\
(9 \%)\end{array}$ & $\begin{array}{l}4 \\
(17 \%)\end{array}$ & $\begin{array}{l}1 \\
(33 \%)\end{array}$ & 0 & 0 & 0 \\
\hline Chills & 0 & 0 & 0 & 0 & 0 & 0 & 0 & $\begin{array}{c}1 \\
(3 \%)\end{array}$ & 0 & 0 & 0 & 0 & 0 & 0 \\
\hline Cough & 0 & 0 & 0 & 0 & 0 & 0 & 0 & $\begin{array}{c}1 \\
(3 \%)\end{array}$ & 0 & 0 & 0 & 0 & 0 & 0 \\
\hline Hyperbilirubinaemia & 0 & $\begin{array}{c}1 \\
(4 \%)\end{array}$ & 0 & 0 & 0 & 0 & 0 & 0 & 0 & 0 & 0 & 0 & 0 & 0 \\
\hline Diarrhoea & 0 & 0 & 0 & 0 & 0 & 0 & 0 & $\begin{array}{c}1 \\
(3 \%)\end{array}$ & 0 & 0 & 0 & 0 & 0 & 0 \\
\hline $\begin{array}{l}\text { Disturbance in } \\
\text { attention }\end{array}$ & 0 & 0 & 0 & 0 & 0 & 0 & 0 & $\begin{array}{c}1 \\
(3 \%)\end{array}$ & 0 & 0 & 0 & 0 & 0 & 0 \\
\hline Dysarthria & 0 & 0 & 0 & 0 & 0 & 0 & 0 & $\begin{array}{c}1 \\
(3 \%)\end{array}$ & 0 & 0 & 0 & 0 & 0 & 0 \\
\hline Dyspnoea & 0 & 0 & 0 & 0 & $\begin{array}{l}1 \\
(2 \%)\end{array}$ & 0 & 0 & $\begin{array}{c}1 \\
(3 \%)\end{array}$ & 0 & 0 & 0 & 0 & 0 & 0 \\
\hline Eczema & 0 & 0 & 0 & 0 & 0 & $\begin{array}{c}1 \\
(2 \%)\end{array}$ & 0 & 0 & 0 & 0 & 0 & 0 & 0 & 0 \\
\hline Oedema & 0 & 0 & 0 & $\begin{array}{c}1 \\
(4 \%)\end{array}$ & 0 & 0 & 0 & 0 & 0 & 0 & 0 & 0 & 0 & 0 \\
\hline $\begin{array}{l}\text { Endocarditis } \\
\text { staphylococcal }\end{array}$ & 0 & 0 & $\begin{array}{c}1 \\
(4 \%)\end{array}$ & 0 & 0 & 0 & 0 & 0 & 0 & 0 & 0 & 0 & 0 & 0 \\
\hline $\begin{array}{l}\text { Gastric varices } \\
\text { haemorrhage }\end{array}$ & 0 & 0 & 0 & 0 & 0 & $\begin{array}{c}1 \\
(2 \%)\end{array}$ & 0 & 0 & 0 & 0 & 0 & 0 & 0 & 0 \\
\hline $\begin{array}{l}\text { Gastroduodenal } \\
\text { ulcer }\end{array}$ & 0 & 0 & $\begin{array}{c}1 \\
(4 \%)\end{array}$ & 0 & 0 & 0 & 0 & 0 & 0 & 0 & 0 & 0 & 0 & 0 \\
\hline Headache & 0 & 0 & 0 & 0 & $\begin{array}{c}1 \\
(2 \%)\end{array}$ & 0 & 0 & 0 & 0 & 0 & 0 & 0 & 0 & 0 \\
\hline $\begin{array}{l}\text { Hepatocellular } \\
\text { carcinoma }\end{array}$ & $\begin{array}{c}1 \\
(4 \%)\end{array}$ & 0 & 0 & $\begin{array}{c}1 \\
(4 \%)\end{array}$ & 0 & 0 & 0 & 0 & 0 & 0 & 0 & 0 & 0 & 0 \\
\hline $\begin{array}{l}\text { Hepatorenal } \\
\text { syndrome }\end{array}$ & 0 & 0 & 0 & $1(4 \%)$ & 0 & 0 & 0 & 0 & 0 & 0 & 0 & 0 & 0 & 0 \\
\hline \multirow[t]{2}{*}{ Dehydration } & 0 & 0 & 0 & 0 & 0 & 0 & 0 & 0 & 0 & 0 & 0 & $\begin{array}{l}1 \\
(20 \%)\end{array}$ & 0 & 0 \\
\hline & & & & & & & & & & & & \multicolumn{3}{|c|}{ (Table 3 continues on next page) } \\
\hline
\end{tabular}




\begin{tabular}{|c|c|c|c|c|c|c|c|c|c|c|c|c|c|c|}
\hline & \multicolumn{4}{|c|}{ Cohort A: before transplantation } & \multicolumn{10}{|c|}{ Cohort B: after transplantation } \\
\hline & \multicolumn{2}{|l|}{ CTP-B } & \multicolumn{2}{|l|}{ CTP-C } & \multicolumn{2}{|c|}{ No cirrhosis } & \multicolumn{2}{|l|}{ CTP-A } & \multicolumn{2}{|l|}{ CTP-B } & \multicolumn{2}{|l|}{ CTP-C } & \multicolumn{2}{|l|}{$\mathrm{FCH}$} \\
\hline & $\begin{array}{l}12 \text { weeks } \\
(n=28)\end{array}$ & $\begin{array}{l}24 \text { weeks } \\
(n=28)\end{array}$ & $\begin{array}{l}12 \text { weeks } \\
(n=25)\end{array}$ & $\begin{array}{l}24 \text { weeks } \\
(n=26)\end{array}$ & $\begin{array}{l}12 \text { weeks } \\
(\mathrm{n}=52)\end{array}$ & $\begin{array}{l}24 \text { weeks } \\
(n=49)\end{array}$ & $\begin{array}{l}12 \text { weeks } \\
(n=34)\end{array}$ & $\begin{array}{l}24 \text { weeks } \\
(n=33)\end{array}$ & $\begin{array}{l}12 \text { weeks } \\
(\mathrm{n}=22)\end{array}$ & $\begin{array}{l}24 \text { weeks } \\
(n=23)\end{array}$ & $\begin{array}{l}12 \text { weeks } \\
(n=3)\end{array}$ & $\begin{array}{l}24 \text { weeks } \\
(n=5)\end{array}$ & $\begin{array}{l}12 \text { weeks } \\
(n=3)\end{array}$ & $\begin{array}{l}24 \text { weeks } \\
(\mathrm{n}=2)\end{array}$ \\
\hline \multicolumn{15}{|c|}{ (Continued from previous page) } \\
\hline Lethargy & 0 & 0 & 0 & 0 & 0 & 0 & 0 & $\begin{array}{c}1 \\
(3 \%)\end{array}$ & 0 & 0 & 0 & 0 & 0 & 0 \\
\hline Pleuritic pain & 0 & 0 & 0 & 0 & 0 & 0 & 0 & $\begin{array}{c}1 \\
(3 \%)\end{array}$ & 0 & 0 & 0 & 0 & 0 & 0 \\
\hline Pyrexia & 0 & 0 & 0 & 0 & 0 & 0 & 0 & $\begin{array}{c}1 \\
(3 \%)\end{array}$ & 0 & 0 & 0 & 0 & 0 & 0 \\
\hline Rash & 0 & 0 & 0 & 0 & 0 & 0 & 0 & 0 & $\begin{array}{c}1 \\
(5 \%)\end{array}$ & 0 & 0 & 0 & 0 & 0 \\
\hline Renal impairment & 0 & 0 & 0 & 0 & 0 & 0 & 0 & 0 & $\begin{array}{l}1 \\
(5 \%)\end{array}$ & 0 & 0 & 0 & 0 & 0 \\
\hline $\begin{array}{l}\text { Staphylococcal } \\
\text { sepsis }\end{array}$ & 0 & 0 & $\begin{array}{c}1 \\
(4 \%)\end{array}$ & 0 & 0 & 0 & 0 & 0 & 0 & 0 & 0 & 0 & 0 & 0 \\
\hline Vision blurred & 0 & 0 & 0 & 0 & 0 & 0 & 0 & $\begin{array}{c}1 \\
(3 \%)\end{array}$ & 0 & 0 & 0 & 0 & 0 & 0 \\
\hline
\end{tabular}

resulted in high rates of SVR in patients with $\mathrm{HCV}$ genotype 1 or 4 and advanced liver disease both before and after liver transplantation, including those with fibrosing cholestatic hepatitis. Patients who underwent transplantation had received at least 11 weeks of antiviral treatment before transplantation, and they maintained HCV RNA less than the LLOQ after transplantation.

Rates of relapse were low and were most frequently seen in non-transplanted patients with decompensated cirrhosis. Among post-transplantation patients with HCV genotype 1 , only one $(<1 \%)$ of 193 relapsed. Additionally, treatment duration did not appear to have an effect on virological response rates for patients with genotype 1 infection, a result that is consistent with the results from SOLAR-1, which also suggested that treatment duration was not associated with any difference in virological response rates in patients with genotype $1 \mathrm{HCV}$.

Overall, similar SVR rates were recorded in patients with and without baseline NS5A resistance-associated variants whether using a $1 \%$ cutoff or $15 \%$ cutoff for both genotype 1 and genotype 4. Future studies should examine if certain patients with specific NS5A resistanceassociated variants or certain combinations of resistanceassociated variants might need 24 weeks of treatment.

Of note, in the European Union, ledipasvir-sofosbuvir plus ribavirin for 24 weeks is indicated for genotype 1 patients with decompensated cirrhosis or those who have undergone liver transplantation or both. The results of both the SOLAR-1 and SOLAR-2 trials suggest that a shortened course of 12 weeks of ledipasvir-sofosbuvir plus ribavirin is probably sufficient treatment for nearly all patients with genotype $1 \mathrm{HCV}$ who have decompensated cirrhosis or with genotype $1 \mathrm{HCV}$ recurrence post-liver transplantation.
Consistent with these conclusions, the 2015 HCV treatment guidelines from the European Association for the Study of the Liver (EASL) recommend 12 weeks of ledipasvir-sofosbuvir plus ribavirin as a first-line option for HCV genotype 1 patients either with decompensated cirrhosis (CTP-B or CTP-C) or with compensated or decompensated cirrhosis after liver transplantation. ${ }^{13}$ The same recommendations have been made by the American Association for the Study of Liver Diseases (AASLD) and the Infectious Diseases Society of America (IDSA). ${ }^{14}$ Recommendations for other regimens for patients with advanced liver disease vary by region. EASL guidelines recommend sofosbuvir plus daclatasvir with weight-based ribavirin for 12 weeks as another first-line option in patients with HCV genotype 1 either with decompensated cirrhosis (CTP-B or CTP-C) or with compensated or decompensated cirrhosis after liver transplantation. ${ }^{13}$

AASLD/IDSA guidelines recommend ombitasvirparitaprevir-ritonavir and dasabuvir plus ribavirin for 24 weeks in liver transplant recipients with genotype 1 $\mathrm{HCV}$, but this regimen is restricted to those without fibrosis or with mild fibrosis. ${ }^{14}$

By contrast with SOLAR-1, which enrolled only five patients with genotype $4 \mathrm{HCV}$, this study enrolled 37 patients with genotype 4 infection. Of these, two were not assessed for efficacy-one was enrolled in error and one received a liver transplant before SVR12 with HCV RNA less than the LLOQ at transplantation-and two additional patients were not assessed for relapse because they died before reaching post-treatment week 12 . Of the remaining 33, three patients (9\%) relapsed, all of whom had decompensated cirrhosis and received 12 weeks of treatment. Thus, although it is reasonable to conclude 
that post-transplantation patients with $\mathrm{HCV}$ recurrence who have compensated disease can receive 12 weeks of treatment, it is challenging to make definitive conclusions about the optimum duration of therapy for genotype 4 patients with decompensated disease based on this still limited number of patients.

Whereas SVR12 rates describe the virological response, changes in MELD and CTP scores describe the clinical response seen in patients. Such changes between start of treatment until 12 weeks after end of treatment were assessed for all decompensated patients (before and after transplantation) who achieved SVR12. Most patients with decompensated cirrhosis had improvements in MELD and CTP scores with viral eradication. Similar improvements in patients with hepatic decompensation were reported in the SOLAR-1 study of HCV antiviral therapy, ${ }^{11}$ in real-world patients with advanced liver disease due to HCV who were treated with regimens including directly acting antivirals, ${ }^{15}$ and in studies of hepatitis B antiviral therapy in patients with hepatitis B-related advanced liver disease, ${ }^{16,17}$ suggesting virological suppression or eradication might improve hepatic function. Thus, successful viral eradication might affect both morbidity and mortality for most patients with decompensated disease. A long-term, 5 -year study is underway to quantify this and to assess whether there is a point of no return (ie, a degree of hepatic impairment for which it is futile to treat). In this study, and in SOLAR-1, patients with CTP scores greater than 12 were excluded due to their high near-term mortality.

For the small subgroup of decompensated patients who are or might be eligible for liver transplantation, however, improving their MELD score and eradicating their HCV infection before transplantation might have a paradoxical consequence. Such patients might lose their eligibility or priority for transplantation as their MELD score decreases; this possibility must be weighed against the time available for completing treatment before transplantation, the possibility of death while waiting for a transplant (or death during transplantation), as well as public health considerations given the general shortage of transplantable liver organs. Future studies will have to show whether HCV eradication is able to improve liver function to such an extent that patients can be removed from the waiting list.

All patients with fibrosing cholestatic hepatitis (5/5), a population that until recently had few treatment options and high mortality, achieved SVR12 after treatment with ledipasvir-sofosbuvir plus ribavirin for 12 or 24 weeks. These results are comparable to those seen when patients with fibrosing cholestatic hepatitis were treated with sofosbuvir plus daclatasvir (100\% SVR12, 15/15) or sofosbuvir plus ribavirin (88\% SVR12, 7/8). ${ }^{18}$

Despite the promising results, this study has several limitations. The small sample sizes do not allow definitive conclusions to be made about the effect of treatment in subgroups. The need for ribavirin, especially among patients without decompensated disease, was not assessed and needs to be defined in future studies. All patients in this study had MELD scores of 25 or below at baseline-in some European countries patients without hepatocellular carcinoma are eligible for liver transplantation only if they have MELD scores higher than 30 . Another limitation was the exclusion of patients with an estimated glomerular filtration rate less than $30 \mathrm{~mL} / \mathrm{min}$. In conclusion, ledipasvir-sofosbuvir plus ribavirin is an effective treatment for patients with HCV genotype 1 or 4 and advanced liver disease, including those with decompensated liver function, before and after liver transplantation. 12 weeks of treatment should become the standard of care for this group of patients. This alloral regimen provides a valuable treatment option for patients who most need one.

\section{Contributors}

MM, DS, EJG, DM, GM, MB, MP, JLC, MP-R, BM, KA, PA, EMY, MC, $\mathrm{MR}, \mathrm{J}-\mathrm{FD}, \mathrm{HVV}, \mathrm{BvH}$, and XF contributed to the recruitment of patients and served as investigators in this study. MM, DS, JD, SA, PSP, DB, JGM, BvH, and XF contributed to the study design. All authors contributed to the writing, review, and data collection or analysis of the report.

\section{SOLAR-2 investigators}

José Luis Calleja, Maria Buti, Xavier Forns, Martín Prieto, Paul Marotta, Eric Yoshida, Vincent Bain, Leslie Lilly, Philip Wong, Bernard Willems, Geoff McCaughan, Peter Angus, Stefan Zeuzem, Guido Gerken,

Christian Trautwein, Michael Manns, Kosh Agarwal, Andrew Bathgate, David Mutimer, Jean-Francois Dufour, Beat Müllhaupt,

Christophe Duvoux, Francois Durand, Didier Samuel, Georges Pageaux, Yves Horsmans, Hans Van Vlierberghe, Massimo Colombo, Mario Rizzetto, Markus Peck-Radosavljevic, Wolfgang Vogel, Edward Gane, Bart van Hoek, Robert de Knegt.

\section{Declaration of interests}

HD-S, JD, SA, PSP, DB, and JGM are employees of Gilead Sciences (Gilead) and hold stock interest in the company. MM reports grants and personal fees from Roche, Bristol-Myers Squibb, Gilead, Boehringer Ingelheim, Novartis, Merck (MSD), Janssen, GlaxoSmithKline, and Biotest, and personal fees from Idenix and Achillion, outside the submitted work. DS reports consultancies with Astellas, Gilead, BMS, LFB, MSD, Novartis, Roche, Biotest, and AbbVie, outside the submitted work. EJG reports that he is a member of the Gilead HCV Global Advisory Board and Gilead Speakers Bureau. GM has grants from Gilead, during the conduct of the study, personal fees from Gilead and AbbVie, outside the submitted work. MB reports personal fees from MSD, Gilead, and Janssen, outside the submitted work. MP is an advisory board member for Gilead, Bristol, AbbVie, and has attended meetings hosted by Janssen Cilag. JLC reports personal fees from Gilead, AbbVie, MSD, BMS, and Janssen. MP-R reports patient fees from Gilead, during the conduct of the study; grants and personal fees from Gilead, AbbVie, BMS, Boehringer Ingelheim, MSD, Roche, and Janssen, outside the submitted work. BM reports grants and personal fees from Gilead, and personal fees from BMS, AbbVie, Janssen, MSD, and Boehringer, outside the submitted work. KA reports personal fees from Achillion, Intercept, GlaxoSmithKline, Janssen, Alnylam, Merck, Novartis, and Astellas, and grants and personal fees from BMS, AbbVie, and Gilead, outside the submitted work. EMY reports grants from Gilead, Janssen, AbbVie, Merck, Hoffman-La Roche, Boeringer Ingelheim, Vertex, Novartis, and personal fees from Gilead Canada, AbbVie Canada, Merck Canada, and Hoffman-La Roche Canada, outside the submitted work. MC is an advisory board member of, and has received speaking and teaching fees from, AbbVie, Gilead, BMS, Roche, Merck, Bayer, Novartis, and Janssen, outside the submitted work. J-FD reports personal fees from Gilead, Merck, BMS, Novartis, AbbVie, and Intercept, outside the submitted work. BvH reports other grants 
from Gilead during the conduct of the study; personal fees for advisory boards from AbbVie, Merck, Janssen, BMS, Falk, and Norgine, outside the submitted work. XF reports grants from Jansen, personal fees from Gilead, and grants and personal fees from AbbVie, outside the submitted work. DM, PA, MR, and HVV declare no competing interests.

\section{Acknowledgments}

We thank the patients and their families, the investigators and study-site personnel, Jennifer King (August Editorial) for providing editorial assistance, and Ravi Grewal, Luke Passler, and Rosalinda Graci for their clinical operations support. This study was supported by Gilead Sciences.

\section{References}

1 van der Meer AJ, Veldt BJ, Feld JJ, et al. Association between sustained virological response and all-cause mortality among patients with chronic hepatitis $\mathrm{C}$ and advanced hepatic fibrosis JAMA 2012; 308: 2584-93.

2 Firpi RJ, Clark V, Soldevila-Pico C, et al. The natural history of hepatitis C cirrhosis after liver transplantation. Liver Transpl 2009; 15: 1063-71.

3 Gilead Sciences. SOVALDI prescribing information. https://www. gilead.com/ /media/Files/pdfs/medicines/liver-disease/sovaldi/ sovaldi_pi.pdf (accessed June 11, 2015).

4 European Medicines Agency. Sovaldi. Summary of opinion. http://www.ema.europa.eu/docs/en_GB/document_library/ Summary_of_opinion_-_Initial_authorisation/human/002798/ WC500155435.pdf (accessed June 11, 2015)

5 Curry MP, Forns X, Chung RT, et al. Sofosbuvir and ribavirin prevent recurrence of $\mathrm{HCV}$ infection after liver transplantation: an open-label study. Gastroenterology 2015; 148: 100-07.

6 Charlton M, Gane E, Manns MP, et al. Sofosbuvir and ribavirin for treatment of compensated recurrent hepatitis $\mathrm{C}$ virus infection after liver transplantation. Gastroenterology 2015; 148: 108-17.

7 Forns X, Charlton M, Denning J, et al. Sofosbuvir compassionate use program for patients with severe recurrent hepatitis $\mathrm{C}$ after liver transplantation. Hepatology 2015; 61: 1485-94.

8 Gilead Sciences. HARVONI prescribing information. http://www. gilead.com/ /media/Files/pdfs/medicines/liver-disease/harvoni/ harvoni_pi.pdf (accessed June 11, 2014).
9 European Medicines Agency. Harvoni. EPAR summary for the public. http://www.ema.europa.eu/docs/en_GB/document_library/ EPAR_-_Summary_for_the_public/human/003850/WC500177997. pdf (accessed June 11, 2015).

10 Bourlière $\mathrm{M}$, Bronowicki J-P, de Ledinghen $\mathrm{V}$, et al. Ledipasvir-sofosbuvir with or without ribavirin to treat patients with HCV genotype 1 infection and cirrhosis non-responsive to previous protease-inhibitor therapy: a randomised, double-blind, phase 2 trial (SIRIUS). Lancet Infect Dis 2015; 15: 397-404.

11 Charlton M, Everson GT, Flamm SL, et al. Ledipasvir and sofosbuvir plus ribavirin for treatment of HCV infection in patients with advanced liver disease. Gastroenterology 2015; 149: 649-59.

12 Merion RM, Schaubel DE, Dykstra DM, Freeman RB, Port FK, Wolfe RA. The survival benefit of liver transplantation. Am J Transplant 2005; 5: 307-13.

13 European Association for the Study of the Liver. EASL recommendations on treatment of hepatitis C 2015. J Hepatol 2015; 63: 199-236.

14 American Association for the Study of Liver Diseases and Infectious Diseases Society of America. Recommendations for testing, managing, and treating hepatitis C. http://www.hcvguidelines.org/ (accessed Jan 23, 2015)

15 Deterding K, Höner Zu Siederdissen C, Port K, et al. Improvement of liver function parameters in advanced HCV-associated liver cirrhosis by IFN-free antiviral therapies. Aliment Pharmacol Ther 2015; 42: 889-901.

16 Schiff ER, Lai C-L, Hadziyannis S, et al. Adefovir dipivoxil therapy for lamivudine-resistant hepatitis B in pre- and post-liver transplantation patients. Hepatology 2003; 38: 1419-27.

17 Schiff E, Lai C-L, Hadziyannis S, et al. Adefovir dipivoxil for wait-listed and post-liver transplantation patients with lamivudine-resistant hepatitis B: final long-term results. Liver Transpl 2007; 13: 349-60.

18 Leroy V, Dumortier J, Coilly A, et al. Efficacy of sofosbuvir and daclatasvir in patients with fibrosing cholestatic hepatitis $\mathrm{C}$ after liver transplantation. Clin Gastroenterol Hepatol 2015; 13: 1993-2001. 